\title{
An Early Aptian coral fauna from the Prebetic (southern Spain)
}

\author{
Hannes LÖSER ${ }^{*}$, Lorenzo VILAS ${ }^{2}$, Consuelo ARIAS ${ }^{2}$, Pedro A. RUIZ-ORTIZ ${ }^{3}$, José M. \\ CASTRO ${ }^{3}$ \& Ginés A. DE GEA ${ }^{3}$
}

${ }^{1}$ Estación Regional del Noroeste, Instituto de Geología, Universidad Nacional Autónoma de México, Blvd. Luis Donaldo Colosio S/N y Madrid, 83250 Hermosillo, Sonora, México; loeser@paleotax.de

${ }^{2}$ Departamento de Estratigrafía-IGEO (CSIC-UCM), Universidad Complutense, 28040 Madrid, Spain; vilasl@geo.ucm.es; ariasc@geo.ucm.es

${ }^{3}$ Departamento de Geología y CEAC Tierra, Universidad de Jaén. 23071, Jaén; paruiz@ujaen.es; jmcastro@ujaen.es; gadegea@ ujaen.es

* Corresponding author

Löser, H., Vilas, L., Arias, C., Ruiz-Ortiz, P.A., Castro, J.M. \& de Gea, G.A. 2013. An Early Aptian coral fauna from the Prebetic (southern Spain). [Una asociación de fauna de corales del Aptiense inferior de la Cordillera Bética]. Spanish Journal of Palaeontology, 28 (2), 193-214.

\begin{abstract}
From the Lower Aptian of the Betic Cordillera a small hermatypic coral fauna is reported. The sampled outcrop is located in the Sierra de la Muela (Prebetic, South Spain). The studied corals come from a carbonate platform megasequence, deposited in the Southern Iberian Palaeomargin during the Early-Late Aptian transition. The coral fauna consists of 19 species in 13 genera belonging to the suborders Archeocaeniina, Faviina, Fungiina, Meandrinina, Microsolenina, and Stylinina. The coral fauna consists of typical and common species. It shows relationship of mainly Barremian to Early Albian faunas from the Western Tethys.
\end{abstract}

Keywords: Shallow marine platform, taxonomy, Cretaceous, western Tethys.

\section{RESUMEN}

Se ha estudiado una asociación de fauna de corales hermatípicos del Aptiense Inferior de la Cordillera Bética. Los afloramientos muestreados se localizan en la Sierra de la Muela (Prebético, sur de España). Los corales investigados se encuentran en una megasecuencia de plataforma carbonatada, que se depositó en el Paleomargen Sudibérico durante el tránsito Aptiense inferior-superior. La fauna de corales está compuesta por 19 especies distribuidas en 13 géneros, que pertenecen a los subórdenes Archeocaeniina, Faviina, Fungiina, Meandrinina, Microsolenina, y Stylinina. La fauna de corales está constituida por especies típicas y comunes, y muestran relación principalmente con faunas del Barremiense a Albiense inferior del Tethys occidental.

Palabras clave: Plataforma marina somera, taxonomía, Cretácico, Tethys occidental. 


\section{INTRODUCTION}

With the Late Barremian the amount of coral faunas increased and reached a world-wide all-Cretaceous record during the Early Aptian (Löser, 2013a). The increase of available faunas was accompanied by an increase on diversity on the species as well as on the genus level. Lower Aptian coral faunas are reported from almost everywhere, but they are comparably less known from the Iberian Peninsula. Considerable large faunas (with more than five species) are reported from Aragón (Bataller, 1949; Bover-Arnal et al., 2012), Catalonia (Bataller, 1937, 1949), Murcia (Morycowa et al., 2001; Löser, 2008; Löser \& Saldaña-Villodre, 2008) and Valencia (Bataller, 1937, 1947), totalling not more than ten localities with more than five coral species. In comparison, Upper Aptian and Lower Albian coral faunas are more abundant and richer in species (Schöllhorn, 1998; Tomás et al., 2008 for the Upper Aptian; the Montmell Fm for the Lower Albian, Moreno Bedmar et al., 2009). The present study, therefore, allows increasing our knowledge on the Iberian Lower Aptian coral faunas and their relationship to other faunas.

\section{STUDY AREA}

The Sierra de la Muela is located in the Murcia province (SE Spain), to the south of the Socovos and to the north of the Moratalla village. This relief represents the easternmost part of the Sierra del Taibilla, characterised by its topographic height and rough landscape. The Sierra de la Muela comprises a geological unit that belongs to the External Zones of the Betic Cordillera (Fig. 1). It is ascribed to the Internal Prebetic Domain, characterised by a stratigraphic succession with continuity between the Jurassic and the Cretaceous (Martín-Chivelet et al., 2002; Vera et al., 2004). The Internal Prebetic palaeogeographic domain represents the distal shallow platform environments developed in the Southern Iberian Palaeomargin during the Mesozoic.

The tectonic structure of the Sierra de la Muela is a large anticline with its northern limb bounded by a fault. This major fault, SW-NE oriented, evolves from listric to reverse, and finally to strike-slip. The southern limb crops out, exposing a 1,000 $\mathrm{m}$ thick succession.

The first detailed geological investigations of the study area started about 40 years ago (Fourcade, 1970; Jerez 1973, 1979a, 1979b; Rodríguez-Estrella, 1979). They comprise regional and mapping studies of the Prebetic units that crop out in the surrounding areas. There is only one paper that focusses on the Sierra de la Muela section (Fourcade et al., 1972). A subsequent paper by Vilas et al. (2001) presented a compilation of the stratigraphy of the region west of the Socovos fault, and established a tectonostratigraphic subdivision.

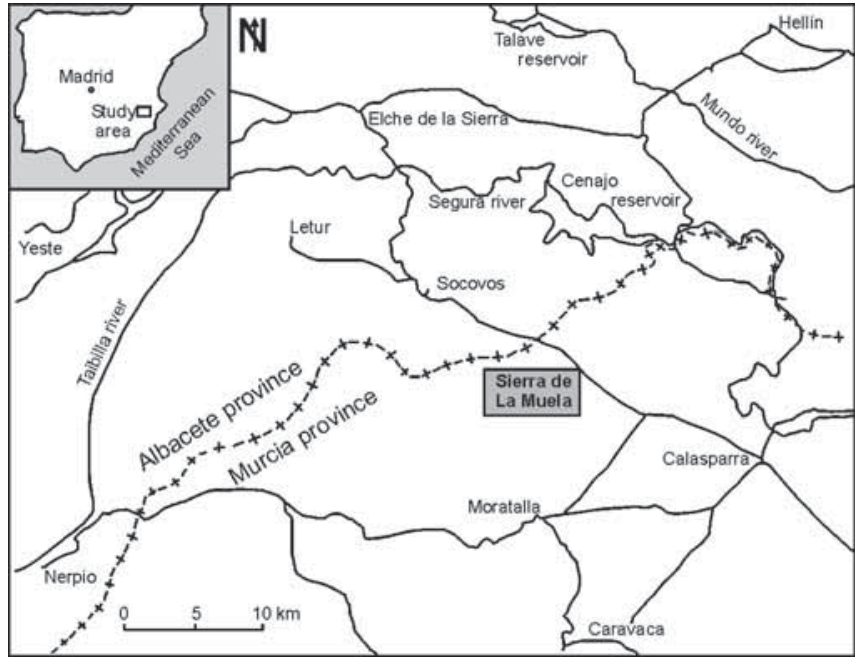

Figure 1. Geographic location of the study area.

The relevance of the stratigraphic characterisation of the Sierra de la Muela is related to its present geological location between first order regional faults, which strongly modify the original location of this sector of the basin during the Mesozoic, especially during the Cretaceous.

\subsection{Stratigraphy}

The stratigraphic section of the Sierra de la Muela embraces a sedimentary succession of Kimmeridgian to Eocene age, and includes several discontinuities, mainly affecting the Upper Cretaceous units (Fig. 2). The Cretaceous succession was subdivided into several tectonostratigraphic units (Martín-Chivelet et al., 2002; Vilas et al., 2003). The Lower Cretaceous presents continuity with the Tithonian limestones up to the Lower Valanginian (K-1 tectonostratigraphic unit), and records the input of the first siliciclastic sediments. After an important discontinuity, marked by a conspicuous hardground surface, the succession continues with the K-2 tectonostratigrahic unit, characterised by shales with abundant ammonites of Late Hauterivian age. Unit K-3 (Upper Barremian), is composed of a succession of grainstones with abundant colonial corals and dasycladaceans limestones, which represent the development of a shallow carbonate platform. The top of unit K-3 is marked by a discontinuity and an abrupt facies change. Unit K-4, of Aptian age, is represented by a 296 m thick succession, with fine terrigenous sediments at the base, and an upper part made of a large variety of shales and carbonate facies. This unit was deposited on a shallow marine platform dominated by carbonate sediments with gastropods, corals and rudists. The upper part of the Cretaceous succession is represented by siliciclastic sediments, limestones and dominant dolomitic units, which belong to the K-5 and following Cretaceous tectonostratigraphic units. 


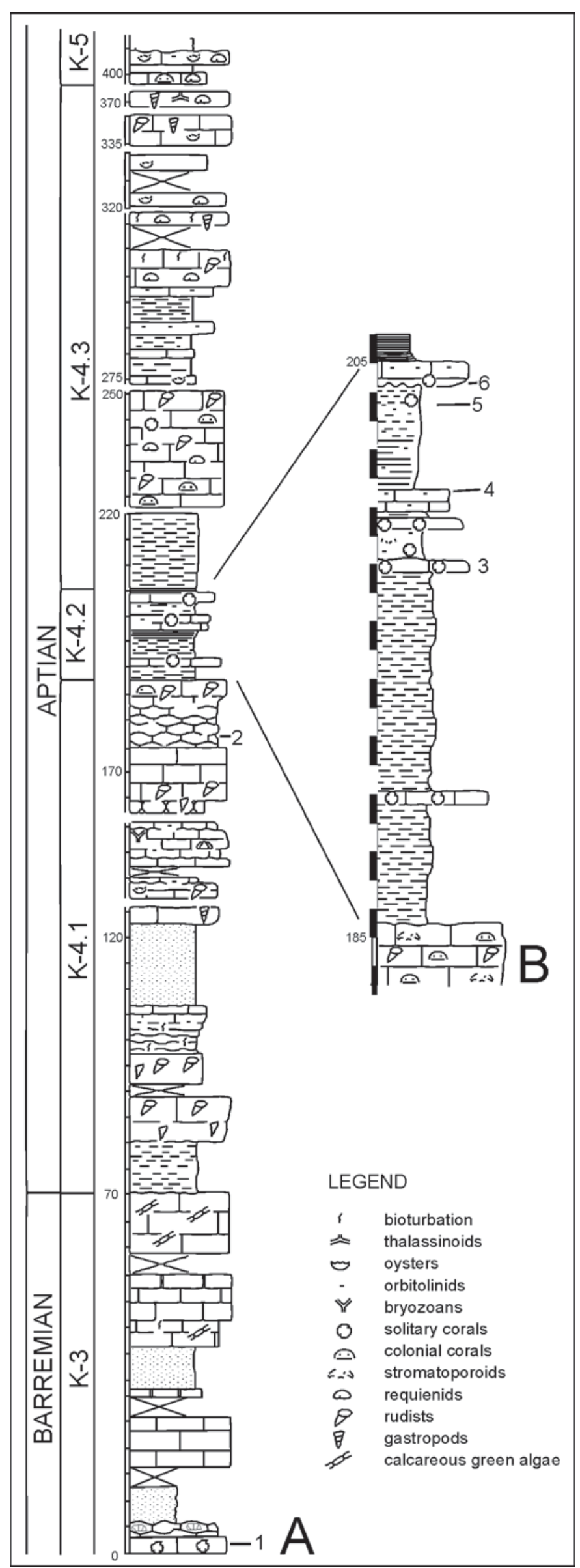

\subsection{The Aptian sediments}

The sedimentary record of the Aptian, that represent the K-4 tectonosedimentary episode (Martín-Chivelet et al., 2002; Vilas et al., 2003) was deposited in a shallow carbonate platform dominated by rudists and corals, with minor siliciclastics episodes. This carbonate platform is hierarchically organised in depositional sequences with a basal terrigenous interval and an upper carbonate unit. Three mega-sequences have been recognised, of Early Aptian (Bedoulian, K-4.1), and Late Aptian (Gargasian, K-4.2 and K-4.3) age, previously defined and characterised in other outcrops of the Prebetic Zone (Vilas et al., 1993, 1998, 2009; Ruiz-Ortiz et al., 2010).

The first mega-sequence (K-4.1), $130 \mathrm{~m}$ thick, is composed of several sequences with terrigenous in the base and carbonates in the upper part, with a retrogradational vertical evolution. The terrigenous facies are dominated by shales in the lower sequences, and by fine sands in the middle part of the mega-sequence. The limestones of the basal sequence contain abundant gastropods, the mid-megasequence carbonates are rich in requienid rudists, and the upper sequences contain nodular limestones with colonial corals. The age of this mega-sequence, deduced from the benthic foraminifera (Orbitolinopsis kiliani Silvestri, 1931, Orbitolinopsis buccifer Arnaud-Vanneau \& Thieuloy, 1972, and Palorbitolina lenticularis Blumenbach, 1805), is Early Aptian (Bedoulian; Masse, pers. comm. 2010).

The second mega-sequence (K-4.2), 15 m thick, is mainly composed of shales with levels rich in corals and orbitolinid foraminifera. The corals studied in this work come from this sequence. The absence of characteristic macro-fauna along with the scarcity of benthic foraminifera prevent an accurate dating at the biozone scale. Nevertheless, the data from the underlying and overlying mega-sequences have led to establishing its age as close to the Early/Late Aptian boundary.

Finally, the third mega-sequence (K-4.3), has a thickness of $137 \mathrm{~m}$ and is partially covered. It is composed of several minor sequences, similar to that from the second mega-sequence, but with a clear predominance of the terrigenous facies, partly responsible for the poor exposure conditions. The carbonate upper division of the sequences evolves from limestones dominated by colonial corals at the base, with minor beds of requienids and radiolitid rudists, to a dominance of a requienid facies in the middle part. The uppermost sequences contain requienids, ostreids and large gastropods, indicating low energy environments.

Figure 2. A) Synthetic stratigraphic section of the Aptian of the Sierra de la Muela. B) Megasequence K-4.2. Numbers indicate the location of the coral bearing beds (see also Table 1). 
The presence of Mesorbitolina sp. in this mega-sequence (Masse, pers. comm. 2010), have led to establishing its age as Late Aptian without more precision.

The sedimentation during the Aptian took place, in general, in low energy environments within an inner carbonate platform, under high sedimentation and subsidence rates. The first mega-sequence records an upwards evolution to more open and higher energy environments. The second mega-sequence is controlled by the input of fine terrigenous sediments and restricted water circulation that prevented a wide development of the coral facies. The third mega-sequence returns to normal proximal mixed-platform environments, and records a vertical evolution from high-energy to more proximal and quiet environments upwards. The corals studied here come mainly from the K-4.2 mega-sequence, where they are mostly embedded in shales.

\section{MATERIALS AND METHODS}

The material comes from six different layers varying much in its coral content (Table 1). The amount of species reflects an abundance of corals and their respective conservation in the various layers. The corals are, for the majority, presented by small colonies (less than $5 \mathrm{~cm}$ ). Their state of preservation is poor and most specimens were only superficially conserved, not allowing the preparation of thin sections, except in transversal, but, not in longitudinal orientation.

Coral slabs and thin sections were examined using a Zeiss STEMI 2000-C stereo microscope. Microphotographs for illustration purposes were obtained using a transparency scanner Epson Perfection V750-M Pro with an optical resolution of 6400 dots per inch. To gain more insight into the intraspecific variation of fossil corals and to obtain a better strategy for comparing species, calicular dimensions one or two thin sections of each species were systematically measured. To achieve statistical significance, the largest number of possible measurements was taken. This number was mainly determined by the size and quality of the thin section and the size of the single calices in relation to the size of the thin sections. Thin sections were measured and values were calculated using the Palaeontological Database System PaleoTax, module PaleoTax/Measure (http://www.paleotax.de/measure). The procedure and statistical background are described in Löser (2012). The obtained morphometric values on the fossils were compared against those on specimens in world wide fossil coral collections using a PaleoTax database (Löser, 2004) on Post-paleozoic corals and associated image database (with 19,000 specimens, about 8,000 illustrated, located in the Estación Regional de Noroeste, Sonora, Mexico).
Table 1. Distribution of the coral species among the various coral bearing beds (numbers refer to Fig. 2B).

\begin{tabular}{|c|c|}
\hline Level & Species \\
\hline 1 & Polyphylloseris simondsi (Wells, 1932) \\
\hline \multirow[t]{2}{*}{2} & Holocystis dupini (d`Orbigny, 1850) \\
\hline & Polyphylloseris simondsi (Wells, 1932) \\
\hline \multirow[t]{18}{*}{3} & Actinaraea bulgarica (Toula, 1877) \\
\hline & Actinaraea robusta Roniewicz, 1966 \\
\hline & ?Complexastrea sp. \\
\hline & Dimorpharaea deickei (Bölsche, 1866) \\
\hline & Dimorpharaea sp. \\
\hline & Diplogyra arasensis (Alloiteau, 1946-47) \\
\hline & Diplogyra casanovai (Reig Oriol, 1994) \\
\hline & Diplogyra sp. \\
\hline & Eugyra arcotica (Stoliczka, 1873) \\
\hline & Holocystis dupini (d'Orbigny, 1850) \\
\hline & Microsolena sp. 1 \\
\hline & Microsolena sp. 2 \\
\hline & Montlivaltia multiformis Toula, 1889 \\
\hline & Plesiosmilia stutzi (Koby, 1896) \\
\hline & Polyphylloseris polymorpha Felix, 1891 \\
\hline & Polyphylloseris simondsi (Wells, 1932) \\
\hline & Stelidioseris japonica (Eguchi, 1951) \\
\hline & Thalamocaeniopsis sp. \\
\hline \multirow[t]{4}{*}{4} & Actinaraea robusta Roniewicz, 1966 \\
\hline & Dimorpharaea deickei (Bölsche, 1866) \\
\hline & Diplogyra casanovai (Reig Oriol, 1994) \\
\hline & Holocystis dupini (d'Orbigny, 1850) \\
\hline \multirow[t]{8}{*}{5} & Actinaraea bulgarica (Toula, 1877) \\
\hline & Dimorpharaea sp. \\
\hline & Dimorphastrea sp. \\
\hline & Diplogyra casanovai (Reig Oriol, 1994) \\
\hline & Holocystis dupini (d’Orbigny, 1850) \\
\hline & Montlivaltia multiformis Toula, 1889 \\
\hline & Plesiosmilia stutzi (Koby, 1896) \\
\hline & Polyphylloseris simondsi (Wells, 1932) \\
\hline 6 & Montlivaltia multiformis Toula, 1889 \\
\hline
\end{tabular}

\section{SYSTEMATIC PALAEONTOLOGY}

Collection abbreviations are as follows: BSPG, Bayerische Staatssammlung für Paläontologie und Geologie, München, Germany; ERNO, Instituto de Geología, Estación Regional de Noroeste, Universidad Nacional Autónoma de México, Hermosillo, Mexico; IGM, Instituto de Geología, Mexico City, Mexico; JCSV, Coll. Saldaña-Villodre, San Vicente del Raspeig, Spain; MGL, Musée Géologique, Lausanne, Switzerland; MGSB, Museo Geológico del Seminario de Barcelona, Spain; TUM, The Tohoku University Museum, Sendai, Japan; UJ, Jagiellonian University, Instytut Nauk 
Geologicznych, Kraków, Poland; UP, Université de Provence, Marseille, France.

The following abbreviations are used describing the dimensions of the corals:

c, (outer) calicular diameter; ccd, distance between calicular centres; cl max, large lumen; cl min, small lumen; cl, calicular diameter (calicular pit); crd, distance of calicular series; crw, width of calicular series; $h$, height of a solitary coral; s, number of radial elements in adult calices; sd, density of radial elements; sdt, density of trabeculae.

The following abbreviations are used describing the statistical values: $n$, number of measurements; min-max, lowest and highest measured value $(\mathrm{mm}) ; \mu$, arithmetic mean (average; $\mathrm{mm}$ ); s, standard deviation ( $\mathrm{mm})$; v, coefficient of variation according to K. Pearson (\%); $\mu \pm \mathrm{s}$, first interval (mm).

The abbreviations used in the synonymy lists follow Matthews (1973): *, earliest valid publication of the species name; ?, the assignation of this description to the species is doubtful (so marked quotations are not reflected in the stratigraphic and palaeobiogeographic distribution); non, the described material does not belong to the species concerned; $\mathrm{p}$, the described material belongs only in part to the species concerned; $v$, the specimen was observed by the author.

Suborder ARCHEOCAENIINA Alloiteau, 1952

Family Actinastraeidae Alloiteau, 1952

Genus Stelidioseris Tomes, 1893

Stelidioseris japonica (Eguchi, 1951)

(Fig. 3a)

Material. BSPG 2003 XX 4156; 1 thin section.

\section{Synonymy.}

*v 1951 Astrocoenia japonica Eguchi, n. sp.; Eguchi, p. 17, Pl. 8, Figs. 7, 8, Pl. 10, Figs. 4, 5

v 1981 Heliocoenia carpathica; Turnšek \& Mihajlovic, p. 11, Pl. 4, Figs. 1-3

v 1994 Actinastrea cf. tourtiensis; Löser, p. 7

v 1995 Actinastrea aequibernensis; Löser \& Raeder, p. 42

v 2006 Actinastrea whitneyi; Löser \& Ferry, p. 475, Figs. 3.4, 3.5

v 2012 Astrocoenia japonica; Löser, p. 383, Fig. 1D

v 2013b Stelidioseris japonica; Löser, p. 91, Figs. 2.1-2.4

\section{Dimensions.}

\begin{tabular}{cclcccc}
\hline (4156) & $\mathbf{n}$ & \multicolumn{1}{c}{$\min -\mathbf{m a x}$} & $\boldsymbol{\mu}$ & $\mathbf{s}$ & $\mathbf{v}$ & $\boldsymbol{\mu} \pm \mathbf{s}$ \\
\hline $\mathrm{cl}$ & 20 & $1.26-1.82$ & 1.57 & 0.16 & 10.1 & $1.40-1.72$ \\
$\mathrm{ccd}$ & 20 & $1.83-3.3$ & 2.54 & 0.4 & 15.7 & $2.13-2.93$ \\
$\mathrm{~s}$ & 24 & & & & & \\
\hline
\end{tabular}

Description. Plocoid colony. Calicular outline polygonal. Septa compact, in cross section externally thick, get slightly thinner towards the centre, septa of the first cycle with swellings (renflements). Symmetry of septa radial and regularly hexameral. Cycles of septa regular. Septal cycles differ in length. First septal cycle reaches to the centre of the calice, further cycles are shorter. Septa of the third cycle occasionally attached to those of the second cycle. Septal distal margin unknown, lateral face smooth (probably due to preservation), inner margin unknown. Pali or paliform lobes absent. All septa of the first cycle are attached to the columella. Costae hardly present, confluent, leaving regular small intercalicinal spaces (lacunes). Synapticulae absent. Columella styliform. Endotheca consists of few dissepiments. Wall present, compact, septothecal. Coenosteum very narrow, consists of costae. Budding unknown.

Occurrence. Cretaceous of Serbia (East Serbia) Planinica. Early Hauterivian (Radiatus zone) of France (Yonne) Fontenoy (BSPG 2003 XX 5064). Late Barremian of France (Ardèche) St. Remèze, Belvedere du Gaud. Aptian of Japan (Kochi-ken) Kami-gun, Birafu-mura, at the former Iwakai Primary School (TUM 65366). Early Aptian of Greece (Viotía) Levadia, Perachorion. Late Aptian of Algeria (Constantine) Sidi R'Gheiss (UP M 6313); Greece (Viotía) Aliartos, Chiarmena; Japan (Iwateken) Miyako-shi, Sakiyama, Hideshima; Japan (Iwate-ken) Shimohei-gun, Iwaizumi-cho, Moshi, Matsushima; Japan (Iwate-ken) Shimohei-gun, Tanohata-mura, Hiraiga; Japan (Iwate-ken) Shimohei-gun, Tanohata-mura, Koikorobe; Japan (Iwate-ken) Shimohei-gun, Taro-cho. Early Albian (Tardefurcata zone) of Spain (Cataluña, Tarragona) Com. Baix Penedès, Mun. Masllorenç, Masarbones (ERNO L-6010). Early Albian of Mexico (Sonora) Municipio Agua Prieta, E San Bernardino Valley, Cordon Caloso (ERNO L-4206). Early Cenomanian (Mantelli zone) of Germany (Nordrhein/Westfalen) Mülheim/Ruhr, Kassenberg. Early Cenomanian of Greece (Kozani) Kozani, Nea Nikopolis (ERNO L-5838).

\footnotetext{
Suborder FAVIINA Vaughan \& Wells, 1943

Family Eugyridae Duncan, 1884

Genus Diplogyra Eguchi, 1936
}

$$
\text { Diplogyra arasensis (Alloiteau, 1946-47) }
$$$$
\text { (Figs 3d-3f) }
$$

Material. BSPG 2003 XX 4154, 2003 XX 7417; 2 thin sections.

\section{Synonymy.}

$*_{\mathrm{v}}$ 1946-47 Eugyra arasensis; Alloiteau, p. 197, Pl. 2, Fig. 4, Text-fig. 2 

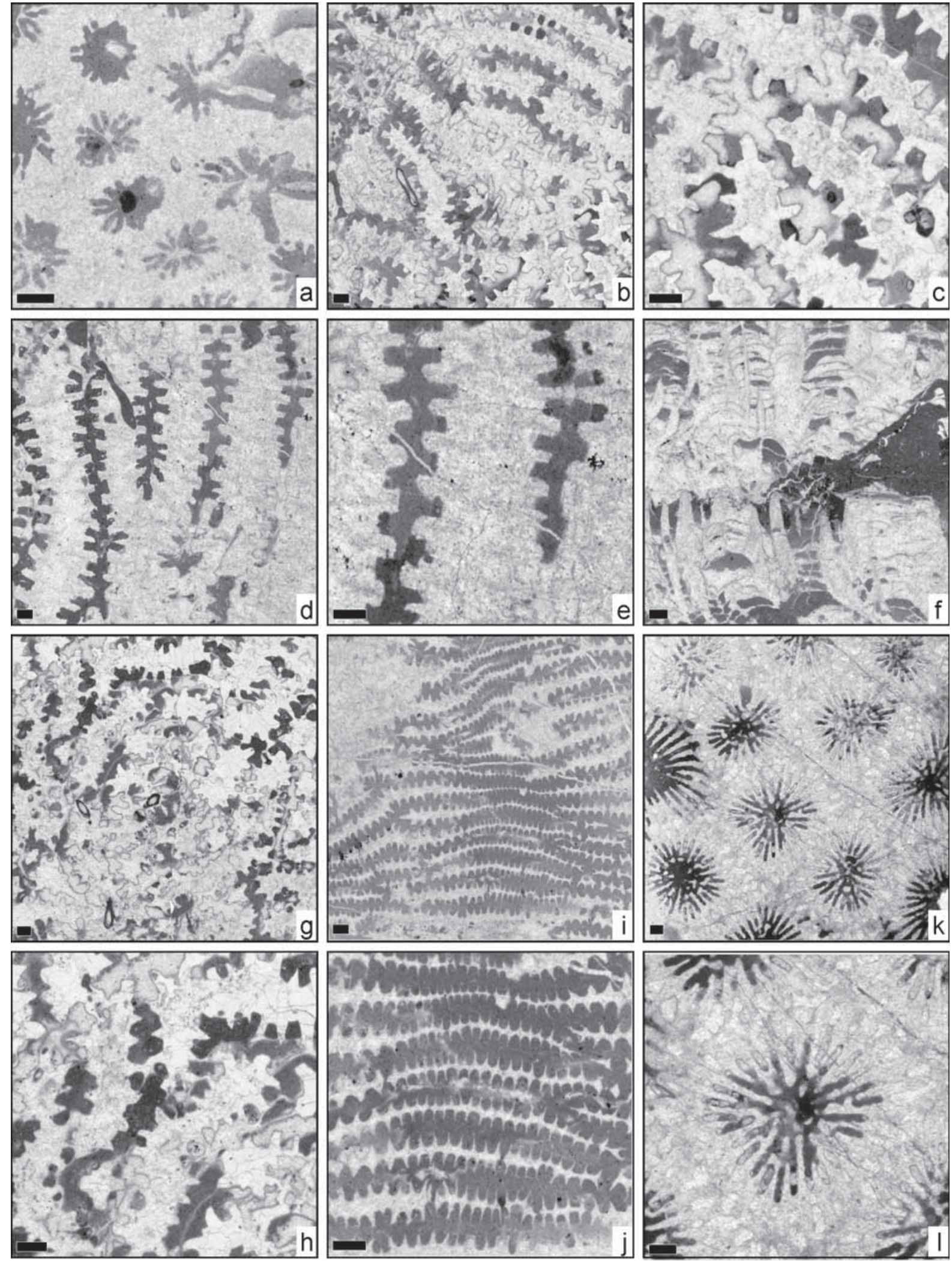

Figure 3. a) Stelidioseris japonica (Eguchi, 1951), (BSPG 2003 XX 4156), transversal thin section. b-c) Diplogyra casanovai (Reig Oriol, 1994), (BSPG 2003 XX 4158); (b) transversal thin section; (c) transversal thin section, detail. d-f) Diplogyra arasensis (Alloiteau, 1946-47), (BSPG 2003 XX 4154); (d) transversal thin section; (e) transversal thin section, detail; (f) longitudinal thin section. g-h) Diplogyra sp., (BSPG 2003 XX 4696); (g) transversal thin section; (h) transversal thin section, detail. i-j) Eugyra arcotica (Stoliczka, 1873), (BSPG 2003 XX 4148); (i) transversal thin section; (j) transversal thin section, detail. k-l) ?Complexastrea sp., (BSPG 2003 XX 4702); (k) transversal thin section; (l) transversal thin section, detail. Scale bar $1 \mathrm{~mm}$. 
1959 Eugyra arasensis; Bataller, p. 21, Text-fig. 784 1994 Eugyra arasensis; Reig Oriol, p. 35, Pl. 1, Fig. a v 1998 Eugyra visesi; Schöllhorn, p. 82, Pl. 18, Figs. 8, 9 v 2008 Diplogyra sp.; Tomás et al., p. 522, Figs. 12C, D v 2013a Diplogyra arasensis; Löser, p. 11, Figs. 4g-i

\section{Dimensions.}

\begin{tabular}{clllllc}
\hline $\mathbf{( 4 1 5 4 )}$ & \multicolumn{1}{c}{$\mathbf{n}$} & $\boldsymbol{m}$ min-max & $\boldsymbol{\mu}$ & \multicolumn{1}{c}{$\mathbf{S}$} & $\mathbf{v}$ & $\boldsymbol{\mu} \pm \mathbf{S}$ \\
\hline $\mathrm{crw}$ & 20 & $1.51-2.74$ & 2.0 & 0.37 & 18.4 & $1.62-2.36$ \\
$\mathrm{crd}$ & 20 & $3.11-4.64$ & 3.85 & 0.5 & 12.9 & $3.35-4.35$ \\
$\mathrm{sd}$ & $10 / 5 \mathrm{~mm}$ & & & & \\
\hline
\end{tabular}

Description. Meandroid colony. Calicular rows long and straight. Calices indistinct. Septa compact, in cross section equal in thickness in the whole septum, but centrally slightly thicker. No septal symmetry. Cycles of septa irregular, but size orders can be distinguished. Septal generations differ in length and thickness. First septal generation reaches $35 \%$ of the calicular diameter, further generations are shorter. Septa free. Septal distal margin unknown, lateral face with fine thorns, inner margin with thorns. Pali or paliform lobes absent. Costae present, non-confluent. Synapticulae absent. Columella absent. Endotheca consists of thin convex tabulae. Wall present, compact, parathecal. Coenosteum medium broad, consists of costae and exothecal dissepiments. Budding intracalicinal, polystomodeal.

Occurrence. Early Hauterivian (Radiatus zone) of France (Yonne) Fontenoy, field $\mathrm{S}$ the junction to Les Merles (BSPG 2003 XX 5289). Early Late Aptian of Spain (Aragón, Huesca) Mun. Espés, Las Aras. Late Aptian of Spain (Valencia, Castellón) Benicasin, La Venta. Latest Aptian of Spain (Cataluña, Lérida) Com. Alt Urgell, Mun. Coll de Nargó, Set Comelles, El Caso section. Early Albian of Mexico (Sonora) Municipio Ures, Cerro de Oro (ERNO L-4938). Early Albian (Mammillatum zone) of France (Aude) Padern, SE Le Crès.

\section{Diplogyra casanovai (Reig Oriol, 1994)}

(Figs 3b-3c)

Material. BSPG 2003 XX 4158, 2003 XX 7419, 2003 XX 7427; 1 thin section.

\section{Synonymy.}

*v 1994 Eugyra casanovai; Reig Oriol, p. 34, Pl. 1, Fig. b, Pl. 2, Fig. a

v 2013a Diplogyra casanovai; Löser, p. 12, Figs. 5ab

\section{Dimensions.}

\begin{tabular}{cllcccc}
\hline $\mathbf{( 4 1 5 8 )}$ & \multicolumn{1}{c}{$\mathbf{n}$} & $\boldsymbol{m i n}-\mathbf{m a x}$ & $\boldsymbol{\mu}$ & $\mathbf{s}$ & $\mathbf{v}$ & $\boldsymbol{\mu} \pm \mathbf{s}$ \\
\hline crw & 25 & $0.82-1.72$ & 1.15 & 0.25 & 21.9 & $0.89-1.39$ \\
crd & 25 & $1.96-2.85$ & 2.45 & 0.27 & 11.1 & $2.17-2.72$ \\
sd & $4 / 2 \mathrm{~mm}$ & & & & \\
\hline
\end{tabular}

Description. Meandroid colony. Calicular rows short and straight. Calices indistinct. Septa compact, in cross section externally thick, getting thinner towards the centre. Symmetry of septa irregular, but two size orders can be distinguished. Cycles of septa subregular. Septal generations differ in length and thickness. First septal generation reaches $35 \%$ of the calicular diameter, further generations are shorter. Septa free. Septal distal margin unknown, lateral face occasionally with small thorns, inner margin smooth. Pali or paliform lobes absent. Costae present, non-confluent. Synapticulae absent. Columella absent. Endotheca consists of thin tabulae. Wall present, compact, parathecal. Coenosteum narrow, consists of costae and exothecal dissepiments. Budding intracalicinal, polystomodeal.

Occurrence. Early Hauterivian (Radiatus zone) of France (Yonne) Gy-l'Evêque, fields SW Gy-l'Evêque (BSPG 2003 XX 6529). Barremian to Early Aptian of Mexico (Puebla) San Juan Raya, Arroyo San Francisco (ERNO L-R10948). Late Barremian to Early Aptian (Lenticularis zone) of Mexico (Sonora) Municipio Ures, Cerro de Oro (ERNO L-4418). Early Aptian (Lenticularis zone) of Spain (Cataluña, Tarragona) Com. Montsià, Mun. Ulldecona, Mas de Comú. Early Albian (Mammillatum zone) of France (Aude) Padern, SE Le Crès.

Diplogyra sp.

(Figs 3g-3h)

Material. BSPG 2003 XX 4696; 1 thin section.

Synonymy.

v 2013a Diplogyra sp.; Löser, p. 12, Figs. 5c-e

Dimensions.

\begin{tabular}{cllcccc}
\hline (4696) & \multicolumn{1}{c}{$\mathbf{n}$} & \multicolumn{1}{c}{$\boldsymbol{\operatorname { m i n }}-\mathbf{m a x}$} & $\boldsymbol{\mu}$ & $\mathbf{s}$ & $\mathbf{v}$ & $\boldsymbol{\mu} \pm \mathbf{s}$ \\
\hline crw & 20 & $0.9-1.27$ & 1.13 & 0.12 & 10.2 & $1.01-1.24$ \\
crd & 20 & $2.07-2.76$ & 2.42 & 0.24 & 9.8 & $2.18-2.65$ \\
sd & $6 / 5 \mathrm{~mm}$ & & & & \\
\hline
\end{tabular}

Description. Meandroid colony. Calicular rows short and curved. Calices indistinct. Septa compact, in cross section externally thick, getting thinner towards the centre. No septal symmetry. Cycles of septa irregular, but size orders can be distinguished. Septal generations differ in 
length and thickness. First septal generation reaches to the centre of the valley, further generations are subsequently shorter. Septa free. Septal distal margin unknown, lateral face rarely with medium size thorns, inner margin smooth. Pali or paliform lobes absent. Costae present, non-confluent. Synapticulae absent. Columella absent. Endotheca consists of thin tabulae. Wall present, compact, parathecal. Coenosteum narrow, consists of costae and exothecal dissepiments. Budding intracalicinal, polystomodeal.

Occurrence. Early Albian (Mammillatum zone) of France (Aude) Padern, SE Le Crès.

Genus Eugyra de Fromentel, 1857

Eugyra arcotica (Stoliczka, 1873)

(Figs 3i-3j)

Material. BSPG 2003 XX 4148; 1 thin section.

\section{Synonymy.}

*v 1873 Stelloria Arcotica; Stoliczka, p. 31, Pl. 5, Fig. 8

\section{Dimensions.}

\begin{tabular}{cllcccc}
\hline $\mathbf{( 4 1 4 8 )}$ & \multicolumn{1}{c}{$\mathbf{n}$} & $\mathbf{m i n}-\mathbf{m a x}$ & $\boldsymbol{\mu}$ & $\mathbf{s}$ & $\mathbf{v}$ & $\boldsymbol{\mu} \pm \mathbf{s}$ \\
\hline crw & 30 & $0.76-1.27$ & 0.99 & 0.12 & 12.5 & $0.86-1.11$ \\
crd & 30 & $0.87-1.63$ & 1.26 & 0.18 & 14.3 & $1.07-1.43$ \\
sd & $16 / 5 \mathrm{~mm}$ & & & & \\
\hline
\end{tabular}

Description. Meandroid colony. Calicular rows long and straight. Calices indistinct. Septa compact, in cross section externally slightly thicker, get slightly thinner towards the centre. No septal symmetry. Most septa reach $40 \%$ of the calicular diameter. Septa free. Septal distal margin unknown, lateral face smooth (probably due to preservation), inner margin smooth. Pali or paliform lobes absent. Costae present, non-confluent. Synapticulae absent. Columella absent. Endotheca consists of thin tabulae. Wall present, compact, septothecal. Coenosteum absent. Budding intracalicinal, polystomodeal.

Occurrence. Early Late Aptian of Algeria (Tebessa) Commune Ouenza, Ouenza Mt (UP 5134 M). Early Albian of USA (Arizona) Cochise County, Douglas, Paul Spur Ridge (ERNO L-4785). Late Albian of India (Tamil Nadu) Perali.

Family Montlivaltiidae Felix, 1900

Genus Complexastrea d'Orbigny, 1849

?Complexastrea sp.

(Figs 3k-31)
Material. BSPG 2003 XX 4702; 1 thin section.

\section{Dimensions.}

\begin{tabular}{cllcccc}
\hline $\mathbf{( 4 7 0 2 )}$ & \multicolumn{1}{c}{$\mathbf{n}$} & $\mathbf{m i n}-\mathbf{m a x}$ & $\boldsymbol{\mu}$ & $\mathbf{s}$ & $\mathbf{c v}$ & $\boldsymbol{\mu} \pm \mathbf{s}$ \\
\hline $\mathrm{cl}$ & 10 & $3.90-5.58$ & 4.58 & 0.56 & 12.4 & $4.01-5.15$ \\
$\mathrm{ccd}$ & 10 & $5.64-7.65$ & 6.61 & 0.60 & 9.1 & $6.00-7.22$ \\
$\mathrm{~s}$ & $40-45$ & & & & & \\
$\mathrm{sd}$ & $5 / 3 \mathrm{~mm}$ & & & & & \\
\hline
\end{tabular}

Description. Astreoid colony. Calicular outline circular. Septa compact, septa and costae in cross section in the wall thick, thinner towards the centre. Symmetry of septa irregular, but three size orders can be distinguished. Cycles of septa subregular. Septal generations differ in length and thickness. First septal generation reaches $35 \%$ of the calicular diameter, further generations are shorter. Septa of younger generations occasionally connected to septa of older ones. Septal distal margin unknown, lateral face occasionally with thorns, inner margin smooth. Paliform lobes irregular on the first two cycles. Costae present, non-confluent. Synapticulae absent. Columella absent. Endotheca not easily observable but dissepiments exist. Wall present, subcompact, parathecal. Coenosteum medium broad, consisting of costae and (?) exothecal dissepiments. Budding extracalicinal.

Remarks. The assignation of the sample to this genus is only tentative. It differs from Complexastrea by the septa that are connected to each other and the formation of paliform lobes. It was not possible to obtain a longitudinal thin section.

Genus Montlivaltia Lamouroux, 1821

Montlivaltia multiformis Toula, 1889

(Figs 4a-4b)

Material. BSPG 2003 XX 4147, 4695, 7410, 7416, 7424; 1 thin section.

Synonymy.

*v 1889 Montlivaltia multiformis; Toula, p. 87, Pl. 6, Fig. 8

v non 1971 Montlivaltia multiformis; Morycowa, p. 75, Pl. 15, Fig. $3[=$ M. hochstetteri]

non 2001 Montlivaltia multiformis; Morycowa et al., p. 137, Figs. 3, 5-12, 13a-e [= M. icaunensis $]$

\section{Dimensions.}

\begin{tabular}{cl}
\hline $\mathbf{( 4 6 9 5 )}$ & \\
\hline $\mathrm{c}$ & $24-28 \mathrm{~mm}$ \\
$\mathrm{~h}$ & $15 \mathrm{~mm}$ \\
$\mathrm{~s}$ & 160 \\
$\mathrm{sd}$ & $8 / 5 \mathrm{~mm}$ \\
\hline
\end{tabular}



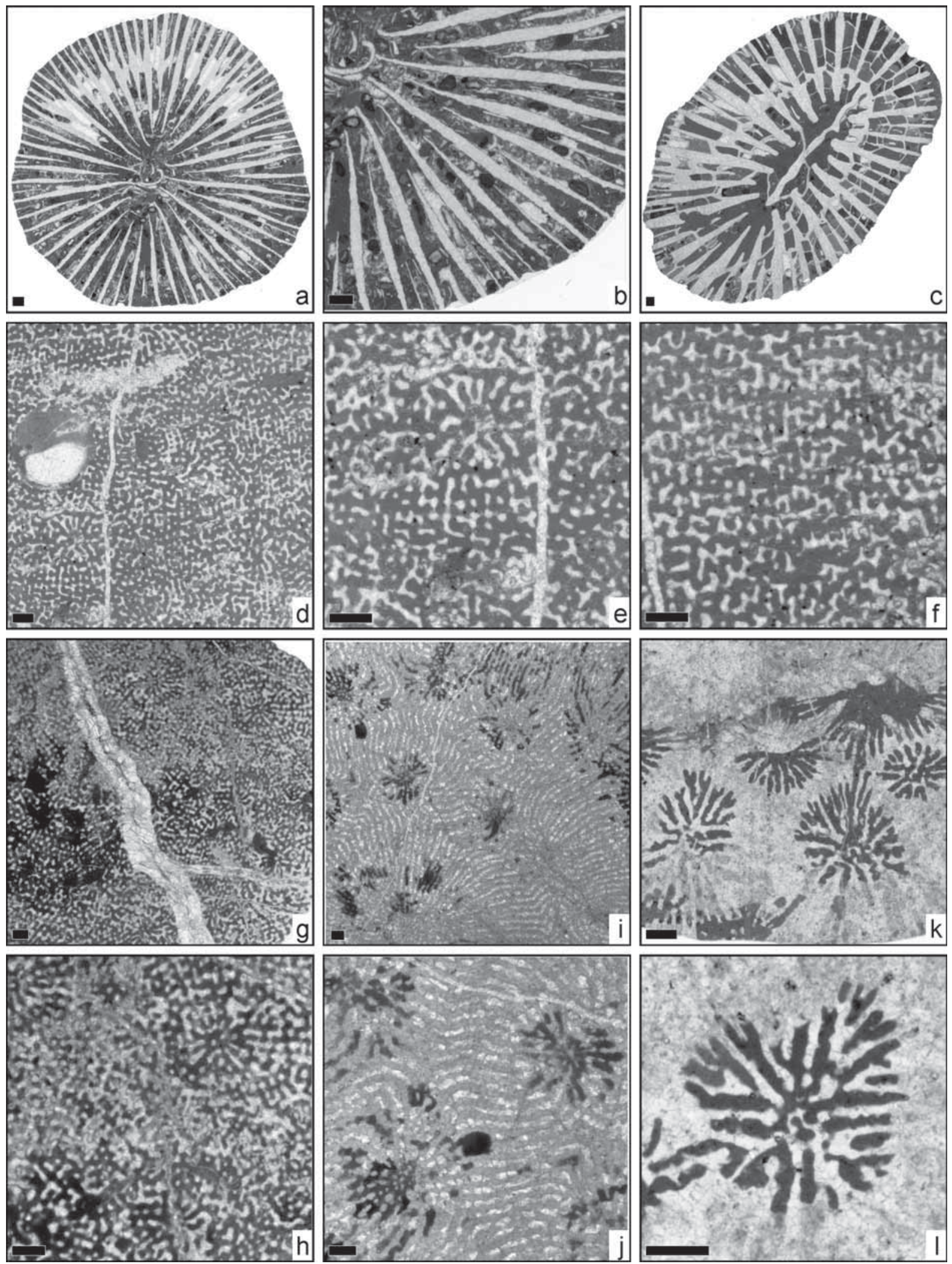

Figure 4. a-b) Montlivaltia multiformis Toula, 1889, (BSPG 2003 XX 4695); (a) transversal thin section; (b) transversal thin section, detail. c) Plesiosmilia stutzi (Koby, 1896), (BSPG 2003 XX 4149), transversal thin section. d-f) Actinaraea bulgarica (Toula, 1877), (BSPG 2003 XX 4157); (d) transversal thin section; (e) transversal thin section, detail; (f) longitudinal thin section. g-h) Actinaraea robusta Roniewicz, 1966, (BSPG 2003 XX 7413); (g) transversal thin section; (h) transversal thin section, detail. i-j) Dimorphastrea sp., (BSPG 2003 XX 7429); (i) transversal thin section; (j) transversal thin section, detail. k-l) Thalamocaeniopsis sp., (BSPG 2003 XX 4698); (k) transversal thin section; (l) transversal thin section, detail. Scale bar $1 \mathrm{~mm}$. 
Description. Solitary cylindric coral. Calicular outline circular. Septa compact. Microstructure of large trabeculae. Septa bicuneiform in cross section. Symmetry of septa irregularly radial. Cycles of septa irregular, but up to four size orders can be distinguished. Septal generations differ in length and thickness. First septal generation reaches close to the centre of the calice, further generations are subsequently shorter. Septa free. Septal distal margin unknown, lateral face with vertical keels, inner margin smooth. Pali or paliform lobes absent. Costae present, surface unknown. Synapticulae absent. Columella absent. Endotheca consists of dissepiments. Wall absent.

Occurrence. Early Hauterivian (Radiatus zone) of France (Yonne) Gy-l'Evêque, fields SE Gy-l'Evêque (BSPG 2003 XX 6103). Barremian of Bulgaria (Veliko Tarnovska oblast) Veliko Tarnovo, Saborena Kanara.

Suborder FUNGIINA Verrill, 1868-70

Family Haplaraeidae Vaughan \& Wells, 1943

Genus Actinaraea d'Orbigny, 1849

\section{Actinaraea bulgarica (Toula, 1877) \\ (Figs 4d-4f)}

Material. BSPG 2003 XX 4152, 4153, 4155, 4157; 2 thin sections.

\section{Synonymy.}

*v 1877 Craticularia bulgarica; Toula, p. 536, Pl. 8, Fig. 7

\section{Dimensions.}

\begin{tabular}{cllcccc}
\hline $\mathbf{( 4 1 5 7 )}$ & \multicolumn{1}{c}{$\mathbf{n}$} & $\mathbf{m i n}-\mathbf{m a x}$ & $\boldsymbol{\mu}$ & $\mathbf{s}$ & $\mathbf{v}$ & $\boldsymbol{\mu} \pm \mathbf{s}$ \\
\hline $\mathrm{ccd}$ & 10 & $3.44-4.78$ & 4.24 & 0.44 & 10.2 & $3.80-4.67$ \\
$\mathrm{~s}$ & $35-45$ & & & & & \\
$\mathrm{sd}$ & $4 / 1 \mathrm{~mm}$ & & & & & \\
$\mathrm{sdt}$ & $4 / 1 \mathrm{~mm}$ & & & & & \\
\hline
\end{tabular}

Description. Thamnasterioid colony with calices arranged in irregular rows. Septa regularly perforated. Microstructure of large trabeculae. Septa in cross section equal in thickness in the whole septum. Symmetry of septa irregular. No regular septal cycles or generations. Half of all septa reach close to the calicular centre. Septa occasionally connected to each other close to the centre of the calice. Septal distal margin coarsely granulated, lateral face with thorns, inner margin smooth. Pali or paliform lobes absent. Costae present, confluent. Synapticulae present, medium common. Columella poorly defined, probably substyliform or sublamellar. Endotheca consists of thin tabulae. Wall absent. Budding extracalicinal, perithecal.
Occurrence. Late Barremian to Early Aptian (Sartousi - Weissi zone) of Germany (Bayern) Allgäuer Helvetikum, Tiefenbach (BSPG 1994 XI 304). Aptian of Mexico (Puebla) San Juan Raya, Barranca Agua del Burro (ERNO R11701). Early Aptian of Serbia (East Serbia) SvetiNikola-Balkan, Kalna.

\section{Actinaraea robusta Roniewicz, 1966 \\ (Figs 4g-4h)}

Material. BSPG $2003 \mathrm{XX} 7405,7413 ; 2$ thin sections.

\section{Synonymy.}

* 1966 Actinaraea robusta; Roniewicz, p. 251, Pl. 25, Fig. 4

v 1998 Actinaraea sp.; Schöllhorn, p. 93, Pl. 25, Fig. 7, 8

\section{Dimensions.}

\begin{tabular}{cllcccc}
\hline $\mathbf{( 7 4 1 3 )}$ & \multicolumn{1}{c}{$\mathbf{n}$} & $\boldsymbol{m i n}-\mathbf{m a x}$ & $\boldsymbol{\mu}$ & $\mathbf{s}$ & $\mathbf{v}$ & $\boldsymbol{\mu} \pm \mathbf{s}$ \\
\hline $\mathrm{ccd}$ & 11 & $4.47-6.31$ & 5.44 & 0.67 & 12.3 & $4.76-6.10$ \\
$\mathrm{~s}$ & $37-40$ & & & & & \\
$\mathrm{sd}$ & $14 / 5 \mathrm{~mm}$ & & & & & \\
\hline
\end{tabular}

Description. Thamnasterioid colony with calices arranged in rows. Septa regularly perforated. Microstructure of large trabeculae. Septa in cross section equal in thickness in the whole septum. Symmetry of septa irregular. No septal cycles. Half of all septa reach close to the calicular centre. Septa occasionally connected to each other close to the centre of the calice. Septal distal margin coarsely granulated, lateral face with thorns, inner margin smooth. Pali or paliform lobes absent. Costae present, confluent. Synapticulae present, abundant. Columella small, substyliform. Endotheca consists of thin tabulae. Wall absent. Coenosteum broad, consists of costae. Budding intracalicinal, polystomodeal and complete.

Occurrence. Late Oxfordian to Early Kimmeridgian of Poland, Sainte-Croix area, Boria. Early Late Aptian of Spain (Cataluña, Lérida) Com. Alt Urgell, Mun. Coll de Nargó, Sta. Fé, Font Bordonera.

Suborder MEANDRININA Alloiteau, 1952

Plesiosmilia group

Remarks. For explanation on this informal group please compare to Löser (2013a).

Genus Plesiosmilia Milaschewitsch, 1876

Plesiosmilia stutzi (Koby, 1896)

(Fig. 4c) 
Material. BSPG $2003 \mathrm{XX} 4149,4151 ; 2$ thin sections.

\section{Synonymy.}

*v 1896 Pleurosmilia Stutzi; Koby, p. 17, Pl. 3, Fig. 1-3 v 1898 Pleurosmilia Villersensis; Koby, p. 89, Pl. 22, Fig. 2-7

v 1905 Peplosmilia Casañasi; Angelis d'Ossat, p. 241, Pl. 17, Fig. 5 a-d

vp 1926 Pleurosmilia hennigi; Dietrich, p. 87, Pl. 7, Fig. 6 a-c

v 1936 Peplosmilia infundibuliformis; Alloiteau, p. 509, Pl. 36, Fig. 7-12

v 1996 Axosmilia kobyi; Baron-Szabo \& Steuber, p. 16, Pl. 8, Fig. 1

v 1996 Axosmilia villersensis; Baron-Szabo \& Steuber, p. 16, Pl. 6, Fig. 6

v 1996 Peplosmilia fromenteli; Baron-Szabo \& Steuber, p. 15, Pl. 7, Fig. 4

\section{Dimensions.}

\begin{tabular}{cll}
\hline & \multicolumn{1}{c}{$(\mathbf{4 1 4 9 )}$} & \multicolumn{1}{c}{$(\mathbf{4 1 5 1 )}$} \\
\hline $\mathrm{c}$ & $22 \times 32 \mathrm{~mm}$ & $21 \times 27 \mathrm{~mm}$ \\
$\mathrm{~s}$ & 90 & 76 \\
$\mathrm{sd}$ & $10 / 10 \mathrm{~mm}$ & $9 / 10 \mathrm{~mm}$ \\
\hline
\end{tabular}

Description. Solitary turbinate coral. Calicular outline elliptical, pit depressed. Septa compact, attenuated to bicuneiform in cross section. Symmetry of septa radial and regularly hexameral. Cycles of septa subregular. Septal cycles differ in length and thickness. First four septal cycles reach close to the center of the calice, further cycles are shorter. Septa free. Septal upper margin smooth, lateral face smooth (probably due to preservation), inner margin smooth. Pali or paliform lobes absent. One septum of the first cycle is attached to the columella. Costae present, smooth on their surface. Synapticulae absent. Columella lamellar. Endotheca consists of dissepiments. Wall absent.

Occurrence. Berriasian of Switzerland (Nidwalden) Urirothstock massif, Bannalp. Late Valanginian to Early Aptian of Tanzania (Tanganyika, Mtwara) Likonde Kitutu plateau, Mikadi. France (Haute-Marne) Morancourt. Barremian of France (Doubs) Morteau, Villers-le-Lac. Late Barremian to Early Aptian (Lenticularis zone) of Mexico (Sonora) Municipio Ures, Cerro de Oro (ERNO L-4333). Aptian of Mexico (Puebla) San Juan Raya, Barranca Agua del Burro (ERNO L-R11702); Greece (Viotía) Arachova. Latest Aptian of Spain (Cataluña, Lérida) Com. Alt Urgell, Mun. Coll de Nargó, Set Comelles, El Caso section (BSPG 2003 XX 4022). Early Albian (Tardefurcata zone) of Spain (Cataluña, Barcelona) Com. Alt Penedés, Mun. Castellví de la Marca, Can Pascual. Early Albian of Mexico (Sonora) Municipio Ures, Cerro de Oro (ERNO L-4355). Early Albian (Mammillatum zone) of France (Aude) Padern, SE Le Crès (SMF 75581). Middle Albian of Mexico (Sonora) Municipio Arivechi, Arivechi, Cerro de las Conchas (ERNO L-4813). Early Cenomanian of Greece (Kozani) Kozani, Nea Nikopolis (BSPG 2003 XX 7440). Late Cenomanian (Plenus zone) of Germany (Sachsen) Dresden-Plauen, Ratssteinbruch, southern quarry (SNSD-MMG 0517).

Suborder MICROSOLENINA Morycowa \& Roniewicz, 1995

Family Leptophylliidae Vaughan, 1905

Genus Dimorphastrea d'Orbigny, 1850

Dimorphastrea sp.

(Figs 4i-4j, 5a-5c)

Material. BSPG 2003 XX 7429, 7430; 5 thin sections.

Synonymy.

vp 1891 Thamnastraea Barcenai; Felix, p. 144, Pl. 22, Fig. 3, Pl. 23, Figs. 7, 7 a

Dimensions.

\begin{tabular}{cllcccc}
\hline $\mathbf{( 7 4 2 9 )}$ & \multicolumn{1}{c}{$\mathbf{n}$} & $\mathbf{m i n}-\mathbf{m a x}$ & $\boldsymbol{\mu}$ & $\mathbf{s}$ & $\mathbf{v}$ & $\boldsymbol{\mu} \pm \mathbf{s}$ \\
\hline $\mathrm{crd}$ & 5 & $6.71-8.52$ & 7.59 & 0.65 & 8.6 & $6.93-8.23$ \\
$\mathrm{ccd}$ & 9 & $5.39-7.15$ & 6.49 & 0.69 & 10.5 & $5.80-7.17$ \\
$\mathrm{~s}$ & $35-40$ & & & & & \\
$\mathrm{sd}$ & $5 / 2 \mathrm{~mm}$ & & & & \\
\hline $\mathbf{( 7 4 3 0 )}$ & $\mathbf{n}$ & $\mathbf{m i n}-\mathbf{m a x}$ & $\boldsymbol{\mu}$ & $\mathbf{s}$ & $\mathbf{v}$ & $\boldsymbol{\mu} \pm \mathbf{s}$ \\
\hline $\mathrm{crd}$ & 10 & $6.35-7.8$ & 7.13 & 0.52 & 7.3 & $6.61-7.65$ \\
$\mathrm{ccd}$ & 15 & $3.33-6.97$ & 5.17 & 1.17 & 22.6 & $4.00-6.34$ \\
$\mathrm{~s}$ & $30-35$ & & & & & \\
$\mathrm{sd}$ & $14 / 5 \mathrm{~mm}$ & & & & \\
\hline
\end{tabular}

Description. Thamnasterioid colony with calices arranged in rows. Septa irregularly perforated, more common at their inner margin. Microstructure of large trabeculae. Septa in cross section externally thick, getting thinner towards the centre. Symmetry of septa irregular. Half of all septa reach close to the calicular centre. Septa occasionally connected to each other close to the centre of the calice. Septal distal margin coarsely granulated, lateral face with pennulae and thorns, inner margin with perforations. Pali or paliform lobes absent. Costae present, confluent. Synapticulae present, medium common. Columella consists of isolated trabeculae or one more solid element. Endotheca consists of few thin tabulae. Wall absent. Budding extracalicinal, perithecal.

Remarks. The material coincides only with one paralectotype of Thamnastraea barcenai, not with the lectotype. 

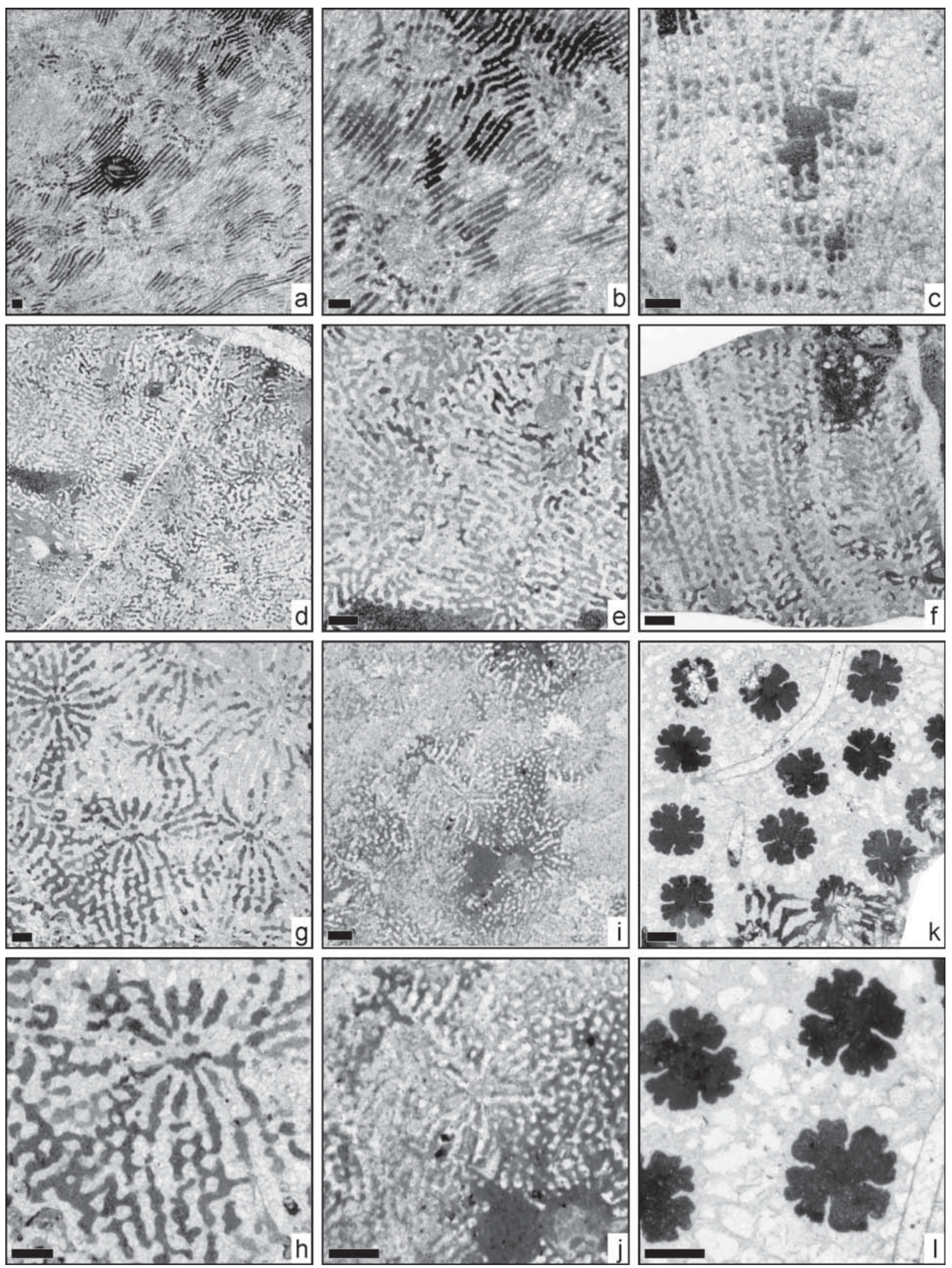

Figure 5. a-c) Dimorphastrea sp., (BSPG 2003 XX 7430); (a) transversal thin section; (b) transversal thin section, detail; (c) longitudinal thin section. d-f) Dimorpharaea deickei (Bölsche, 1866), (BSPG 2003 XX 4692); (d) transversal thin section; (e) transversal thin section, detail; (f) longitudinal thin section. g-h) Microsolena sp., (BSPG 2003 XX 4150); (g) transversal thin section; (h) transversal thin section, detail. i-j) Polyphylloseris polymorpha Felix, 1891, (BSPG 2003 XX 4159); (i) transversal thin section; (j) transversal thin section, detail. k-l) Holocystis dupini (d'Orbigny, 1850), (BSPG 2003 XX 4142); (k) transversal thin section; (l) transversal thin section, detail. Scale bar $1 \mathrm{~mm}$. 
Occurrence. Barremian of Mexico (Puebla) Tehuacán, San Antonio Texcala. Early Albian (Mammillatum zone) of France (Aude) Padern, SE Le Crès (SMF 75568). Early Cenomanian (Saxbii - Dixoni zone) of Germany (Sachsen) Meißen-Zscheila, Trinitatis church (ERNO L-6167). Early Cenomanian (Dixoni zone) of Spain (Cantabria, Santander) Cobreces, Luaña playa (BSPG 2007 V 1). Late Cenomanian (Plenus zone) of Germany (Sachsen) DresdenPlauen, Ratssteinbruch, southern quarry (ERNO L-6137).

Genus Thalamocaeniopsis Alloiteau, 1954

\section{Thalamocaeniopsis sp.}

(Figs 4k-4l)

Material. BSPG 2003 XX 4698, 7407; 1 thin section.

\section{Synonymy.}

v 1926 Isastraea cf. geometrica; Dietrich, p. 79, Pl. 7, Fig. 3

v 2002 Latiastrea paronai; Morycowa \& MarcopoulouDiacantoni, p. 53, Figs. 34a-e, 35a

\section{Dimensions.}

\begin{tabular}{clllllc}
\hline $\mathbf{( 4 6 9 8 )}$ & \multicolumn{1}{c}{$\mathbf{n}$} & \multicolumn{1}{c}{ min-max } & $\boldsymbol{\mu}$ & \multicolumn{1}{c}{$\mathbf{s}$} & \multicolumn{1}{c}{$\mathbf{v}$} & $\boldsymbol{\mu} \pm \mathbf{s}$ \\
\hline $\mathrm{cl} \mathrm{min}$ & 10 & $1.95-3.09$ & 2.5 & 0.41 & 16.4 & $2.09-2.91$ \\
$\mathrm{cl} \mathrm{max}$ & 10 & $2.77-3.93$ & 3.49 & 0.34 & 9.8 & $3.14-3.82$ \\
$\mathrm{ccd}$ & 20 & $3.2-4.96$ & 3.91 & 0.54 & 13.7 & $3.37-4.44$ \\
$\mathrm{~s}$ & $45-50$ & & & & & \\
$\mathrm{sd}$ & $4 / 1 \mathrm{~mm}$ & & & & \\
\hline
\end{tabular}

Description. Cerioid colony. Calicular outline polygonal. Septa perforated at their inner margin, in cross section externally thick, getting thinner towards the centre. Symmetry of septa irregular. Cycles of septa irregular, but size orders can be distinguished. Septal generations differ in length and thickness. First septal generation reaches $40 \%$ of the (shorter) calicular diameter, further generations are shorter. Septa of younger generations often connected to septa of older ones. Septal distal margin unknown, lateral face with pennulae and thorns, inner margin with perforations. Pali or paliform lobes absent. Costae absent. Synapticulae present, occasional, mainly in the space between calices. Columella consists of isolated trabeculae or one more solid element. Endotheca unknown. Wall present, compact, septothecal, with synapticulae. Coenosteum absent. Budding extracalicinal.

Occurrence. Late Barremian of Poland (Malopolskie, Tarnów) Tarnów, Trzemesna (UJ nn). Early Aptian (Weissi Furcata zone) of Tanzania (Tanganyika, Mtwara) Nambawala plateau, Pilepile. Aptian to Cenomanian of Greece (Fokída) Agrostylia. Early Albian of Mexico (Sonora) Municipio
Ures, Cerro de Oro (ERNO L-4914). Late Cenomanian (Plenus zone) of Germany (Sachsen) Dresden-Plauen, Ratssteinbruch, southern quarry (BSPG 2009 XVII 61).

Family Microsolenidae Duncan, 1884

Genus Dimorpharaea de Fromentel, 1861

Dimorpharaea deickei (Bölsche, 1866)

(Figs 5d-5f)

Material. BSPG 2003 XX 4692, 7401, 7402, 7403, 7411, 7415; 2 thin sections.

\section{Synonymy.}

* 1877 Dimorphastraea Deikei; Bölsche, p. 71

v 1994 Dimorpharaea deickei; Löser, p. 46, Text-figs. 38-40, Pl. 1, Fig. 2, Pl. 3, Figs. 1-4

v 2003 Thamnoseris delorenzoi; Baron-Szabo \& González León, p. 219, Fig. 8D

v 2008 Dimorpharaea cf. manchacanensis; Tomás, Löser \& Salas Roig, p. 528, Figs. 14G-H

v 2009 Microsolena parva; Morycowa \& Masse, p. 107, Fig. 7

v 2013a Dimorpharaea deickei; Löser, p. 26, Figs. 9j-1

Dimensions.

\begin{tabular}{clccccc}
\hline (4692) & \multicolumn{1}{c}{$\mathbf{n}$} & $\mathbf{m i n}-\mathbf{m a x}$ & $\boldsymbol{\mu}$ & $\mathbf{s}$ & $\mathbf{v}$ & $\boldsymbol{\mu} \pm \mathbf{s}$ \\
\hline $\mathrm{ccd}$ & 10 & $3.49-4.75$ & 4.17 & 0.51 & 12.1 & $3.66-4.67$ \\
$\mathrm{crd}$ & 10 & $3.91-4.88$ & 4.39 & 0.35 & 7.8 & $4.04-4.73$ \\
$\mathrm{~s}$ & $25-35$ & & & & & \\
$\mathrm{sd}$ & $7 / 2 \mathrm{~mm}$ & & & & & \\
\hline
\end{tabular}

Description. Thamnasterioid colony with calices arranged in sub-regular rows. Calicular centres slightly depressed. Septa irregularly perforated, in cross section equal in thickness in the whole septum. No septal symmetry. Half of all septa reach to the calicular centre. Septa occasionally connected to each other close to the centre of the calice. Septal distal margin unknown, lateral faces with pennulae. Pali or paliform lobes absent. Synapticulae abundant. Columella poorly defined, probably some isolated trabeculae. Endotheca with very few thin tabulae. Wall absent. Budding intracalicinal, polystomodeal and complete.

Remarks. The specimens show a lower grade of perforation than usually in this genus. It is possible that the perforations are just very small.

Occurrence. Late Barremian to Early Aptian (Lenticularis zone) of Mexico (Sonora) Municipio Ures, Cerro de Oro. Aptian of Mexico (Puebla) San Juan Raya (IGM 9226). Early Aptian (Tuarkyricus - Weissi zone) of 
France (Vaucluse) Sault, Moulin d'Aumage. Late Aptian of Spain (Valencia, Castellón) Benicasin, La Venta. Early Albian of Mexico (Sonora) Municipio Naco, quarry E Naco (ERNO L-4961). Early Albian (Mammillatum zone) of France (Aude) Padern, SE Le Crès. Cenomanian of Germany (Nordrhein/Westfalen) Mülheim/Ruhr, Dimbecke. Early Cenomanian (Mantelli zone) of Germany (Nordrhein/Westfalen) Mülheim/Ruhr, Kassenberg. Early Cenomanian (Dixoni zone) of Spain (Cantabria, Santander) Cobreces, Luaña playa/R1R2 (BSPG 2007 V 198).

\section{Dimorpharaea sp.}

(Fig. 6a)

Material. BSPG 2003 XX 4143, 4144, 4694; 1 thin section.

Synonymy.

1985 Dimorpharaea cf. barcenai; Sikharulidze, p. 44, pl. 20 , fig. 3

\section{Dimensions.}

\begin{tabular}{cl}
\hline (4694) & \\
\hline $\mathrm{crd}$ & $6.3-6.9 \mathrm{~mm}$ \\
$\mathrm{cdw}$ & $6.5-7.5 \mathrm{~mm}$ \\
$\mathrm{~s}$ & 40 \\
$\mathrm{sd}$ & $6 / 2 \mathrm{~mm}$ \\
$\mathrm{sdt}$ & $6 / 2 \mathrm{~mm}$ \\
\hline
\end{tabular}

Description. Thamnasterioid colony with calices arranged in rows. Septa regularly perforated. Microstructure of large trabeculae. Septa in cross section equal in thickness in the whole septum. No septal symmetry. Half of all septa reach close to the calicular centre. Septa occasionally connected to each other close to the centre of the calice. Septal distal margin with large regular granules, lateral face with pennulae, inner margin smooth. Pali or paliform lobes absent. Costae present, confluent, with thick granules on their surface. Synapticulae present, abundant, mainly in the space between calices. Columella consists of a group of isolated trabeculae. Endotheca unknown. Wall absent. Budding intracalicinal, polystomodeal and complete.

Occurrence. Hauterivian of Georgia (Imereti) Mukhura. Early Hauterivian (Radiatus zone) of France (Yonne) Fontenoy (BSPG 2003 XX 5678). Early Albian of Mexico (Sonora) Municipio Ures, Cerro de Oro (ERNO L-4357). Early Coniacian of Spain (Cataluña, Lérida) Pallars Jussa, Congost d'Erinya (BSPG 2007 IV CE C18).

Genus Microsolena Lamouroux, 1821
Material. BSPG 2003 XX 4150; 1 thin section.

Synonymy.

v 1997 Microsolena distefanoi; Baron-Szabo, p. 82, Pl. 13, Fig. 5

v 1999 Microsolena distefanoi; Baron-Szabo \& González León, p. 486, Fig. $5 f$

\section{Dimensions.}

\begin{tabular}{cllcccc}
\hline $\mathbf{( 4 1 5 0 )}$ & \multicolumn{1}{c}{$\mathbf{n}$} & $\mathbf{m i n}-\mathbf{m a x}$ & $\boldsymbol{\mu}$ & $\mathbf{s}$ & $\mathbf{v}$ & $\boldsymbol{\mu} \pm \mathbf{s}$ \\
\hline $\mathrm{ccd}$ & 17 & $4.51-6.86$ & 5.64 & 0.67 & 11.9 & $4.96-6.31$ \\
$\mathrm{~s}$ & $32-36$ & & & & & \\
$\mathrm{sd}$ & $9 / 5 \mathrm{~mm}$ & & & & & \\
$\mathrm{sdt}$ & $4 / 1 \mathrm{~mm}$ & & & & & \\
\hline
\end{tabular}

Description. Thamnasterioid colony. Septa regularly perforated. Microstructure of large trabeculae. Septa in cross section equal in thickness in the whole septum. No septal symmetry. Half of all septa reach to the calicular centre. Septa occasionally connected to each other. Septal distal margin with large regular granules, lateral face with pennulae, inner margin with perforations. Pali or paliform lobes absent. Synapticulae present, abundant. Columella poorly defined, probably some isolated trabeculae. Endotheca unknown. Wall absent. Budding intracalicinal, polystomodeal and complete.

Occurrence. Early Hauterivian (Radiatus zone) of France (Yonne) Fontenoy (BSPG 2003 XX 5669). Early Albian of Mexico (Sonora) Municipio Ures, Cerro de Oro (ERNO L-4907). Middle Albian of Mexico (Sonora) Municipio San Pedro de la Cueva, Tepache, Lampazos area. Late Turonian to Early Coniacian of Austria (Tirol) Brandenberg, Haidach.

\section{Microsolena sp. 2}

(Figs 6b-6c)

Material. BSPG 2003 XX 4701; 1 thin section.

\section{Dimensions.}

\begin{tabular}{cl}
\hline $\mathbf{( 4 7 0 1 )}$ & \\
\hline $\mathrm{crd}$ & $5.2-6.5$ \\
$\mathrm{ccd}$ & $4.4-6.1$ \\
$\mathrm{~s}$ & 30 \\
$\mathrm{sd}$ & $6 / 2 \mathrm{~mm}$ \\
$\mathrm{sdt}$ & $4 / 1 \mathrm{~mm}$ \\
\hline
\end{tabular}

Description. Thamnasterioid colony. Septa regularly perforated. Microstructure of large trabeculae. Septa in cross section equal in thickness in the whole septum. No septal symmetry. Half of all septa reach to the calicular centre. Septa occasionally connected to each other close 

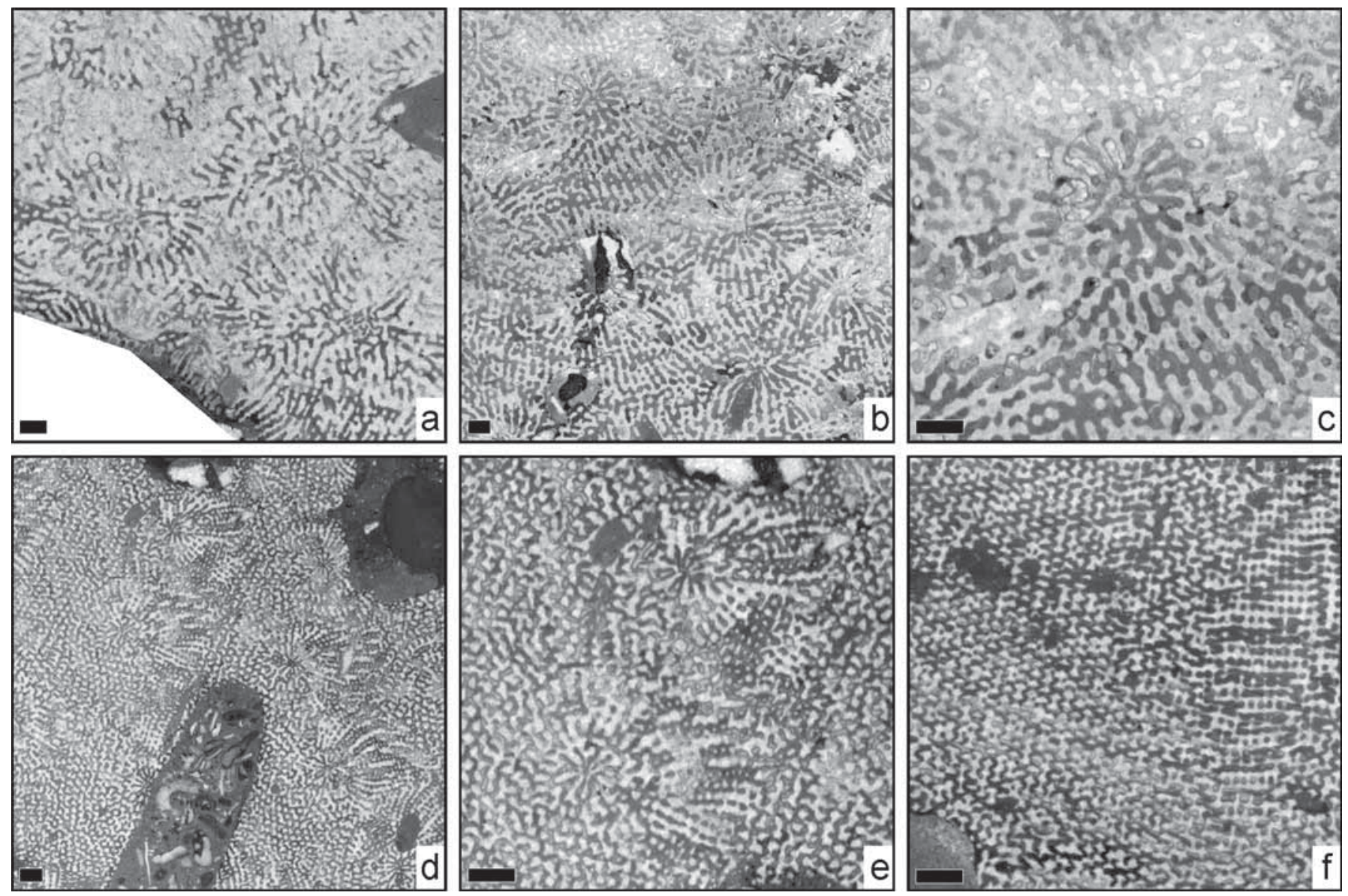

Figure 6. a) Dimorpharaea sp., (BSPG 2003 XX 4694), transversal thin section. b-c) Microsolena sp., (BSPG 2003 XX 4701 ); (b) transversal thin section; (c) transversal thin section, detail. d-f) Polyphylloseris simondsi (Wells, 1932), (BSPG 2003 XX 4697), (d) transversal thin section; (e) transversal thin section, detail; (f) longitudinal thin section. Scale bar $1 \mathrm{~mm}$.

to the centre of the calice. Septal distal margin with large regular granules, lateral face with pennulae, inner margin with perforations. Pali or paliform lobes absent. Costae present, confluent, with thick granules on their surface. Synapticulae abundant. Columella consists of isolated trabeculae or one more solid element. Endotheca not easily observable but dissepiments exist. Wall absent. Budding extracalicinal, perithecal.

Occurrence. Early Hauterivian (Radiatus zone) of France (Yonne) Fontenoy (BSPG 2003 XX 6083) and Leugny (BSPG 2003 XX 5983). Late Barremian to Early Aptian (Lenticularis zone) of Mexico (Sonora) Municipio Ures, Cerro de Oro (ERNO L-4323). Early Aptian of Spain (Murcia) Jumilla, Solano del Sopalmo (JCSV C245); Greece (Viotía) Levadia, Perachorion (BSPG 2003 XX 1870). Early Coniacian of Spain (Cataluña, Lérida) Pallars Jussa, Congost d'Erinya (BSPG 2007 IV CE IB1).

Genus Polyphylloseris de Fromentel, 1857

Polyphylloseris polymorpha Felix, 1891

(Figs 5i-5j)
Material. BSPG 2003 XX 4159; 1 thin section.

\section{Synonymy.}

*v 1891 Polyphylloseris polymorpha; Felix, p. 143, Pl. 22, Figs. 4, 6, 6 a

v 1989 Polyphylloseris polymorpha; Carreño, Perrilliat \& Gonzáles-Arreola, p. 97, Fig. 34k

v 2005 Polyphylloseris sp.; Götz et al., p. 878, Fig. $8 \mathrm{~K}$

v 2006b Polyphylloseris polymorpha; Löser, p. 47, Fig. 4J

v 2009 Polyphylloseris polymorpha; Löser et al., p. 345, Figs. 7.1-5

\section{Dimensions.}

\begin{tabular}{cllcccc}
\hline $\mathbf{( 4 1 5 9 )}$ & \multicolumn{1}{c}{$\mathbf{n}$} & $\mathbf{m i n}-\mathbf{m a x}$ & $\boldsymbol{\mu}$ & $\mathbf{s}$ & $\mathbf{v}$ & $\boldsymbol{\mu} \pm \mathbf{s}$ \\
\hline $\mathrm{c}$ & 12 & $2.03-2.67$ & 2.43 & 0.22 & 8.9 & $2.21-2.65$ \\
$\mathrm{ccd}$ & 20 & $2.67-5.2$ & 3.85 & 0.74 & 19.2 & $3.10-4.59$ \\
$\mathrm{~s}$ & $40-50$ & & & & & \\
$\mathrm{sd}$ & $2 / 5 \mathrm{~mm}$ & & & & & \\
\hline
\end{tabular}


Description. Plocoid colony. Calicular outline circular. Calices slightly elevated. Septa regularly perforated. Microstructure of large trabeculae. Septa in cross section equal in thickness in the whole septum. No septal symmetry. Half of all septa reach to the calicular centre. Septa generally free, rarely connected to each other in the centre of the calice. Septal distal margin with large regular granules, lateral face with pennulae, inner margin with perforations. Pali or paliform lobes absent. Costae present, confluent or not. Synapticulae abundant. Columella poorly defined, probably some isolated trabeculae. Endotheca absent. Wall absent. Coenosteum broad, consists of costae. Budding extracalicinal, perithecal.

Occurrence. Valanginian of Switzerland (Vaud) Arzier (MGL nn). Hauterivian of Spain (Valencia, Castellón) La Avellà, Catí. Jamaica (Saint Catharine) Benbow Inlier, Copper. Barremian of Mexico (Puebla) Tehuacán, San Antonio Texcala. Early Late Albian (Inflatum zone) of Spain (Valencia, Alicante) Sierra de Llorençá (MGSB 74319). Early Cenomanian (Dixoni zone) of Spain (Cantabria, Santander) Cobreces, Luaña playa/R1R2 (BSPG 2007 V 11-4-6).

\section{Polyphylloseris simondsi (Wells, 1932)}

(Figs 6d-6f)

Material. BSPG 2003 XX 4139, 4141, 4146, 4697, 7404, 7409, 7412, 7418, 7421; 2 thin sections.

\section{Synonymy.}

vp 1926 Camptodocis brancai; Dietrich, p. 99, Pl. 5, Fig. 4, Pl. 7, Fig. 11, Pl. 8, Fig. 3

*v 1932 Polyphyllastrea simondsi; Wells, p. 250, Pl.

36, Figs. 4, 5, Pl. 39, Fig. 6

1943 Polyphylloseris simondsi; Vaughan \& Wells, Pl.

21, Fig. 3

1956 Polyphylloseris simondsi; Wells, p. 392, Fig. 285.4

v 1963 Polyphylloseris conophora; Reyeros Navarro,

p. 15, Pl. 4, Figs. 1-6

v 1974 Ovalastraea turbinata; Turnšek \& Buser, p. 22,

37, Pl. 11, Fig. 3

v 2012 Polyphylloseris simondsi; Bover Arnal et al., p. 58 , Figs. $10 \mathrm{~S}-\mathrm{T}$

\section{Dimensions.}

\begin{tabular}{cllcccc}
\hline $\mathbf{( 4 6 9 7 )}$ & \multicolumn{1}{c}{$\mathbf{n}$} & $\mathbf{m i n}-\mathbf{m a x}$ & $\boldsymbol{\mu}$ & $\mathbf{s}$ & $\mathbf{v}$ & $\boldsymbol{\mu} \pm \mathbf{s}$ \\
\hline $\mathrm{c}$ & 20 & $1.96-2.61$ & 2.37 & 0.21 & 8.8 & $2.16-2.58$ \\
$\mathrm{ccd}$ & 25 & $3.08-6.5$ & 4.65 & 0.97 & 20.7 & $3.68-5.61$ \\
$\mathrm{~s}$ & $50-70$ & & & & & \\
$\mathrm{sd}$ & $5 / 1 \mathrm{~mm}$ & & & & \\
$\mathrm{sdt}$ & $5 / 1 \mathrm{~mm}$ & & & & \\
\hline
\end{tabular}

Description. Plocoid colony. Calicular outline circular. Calices slightly elevated. Septa irregularly perforated, costae more regular perforated. Microstructure of large trabeculae. Septa in cross section equal in thickness in the whole septum. No septal symmetry. Septal generations differ in length. Half of all septa reach to the calicular centre. Septa occasionally connected to each other in the centre of the calice. Septal distal margin with large regular granules, lateral face with pennulae, inner margin with perforations. Pali or paliform lobes absent. Costae present, confluent or not, with thick granules on their surface. Synapticulae abundant. Columella poorly defined, probably formed by some isolated trabeculae. Endotheca consists of thin tabulae. Wall absent. Coenosteum broad, consisting of costae. Budding extracalicinal, perithecal.

Occurrence. Aptian of Mexico (Puebla) San Juan Raya; Slovenia (West Slovenia) Banskja Planota, Osojnica. Early Aptian (Weissi - Furcata zone) of Tanzania (Tanganyika, Mtwara) Kiturika Mts, Machemba, Noro. Early Aptian (Furcata zone) of Spain (Aragón, Teruel) Mun. Miravete de la Sierra, Com. Maestrazgo, El Morrón. Early Late Aptian of USA (Texas) Hays County. Early Albian (Tardefurcata zone) of USA (Texas) Hays County, Blanco River, Pleasant Valley Crossing.

Suborder STYLININA Alloiteau, 1952

Family Cyathophoridae Duncan, 1884

Genus Holocystis Lonsdale, 1849

Holocystis dupini (d`Orbigny, 1850)

(Figs 5k-5l)

Material. BSPG 2003 XX 4140, 4142, 4145, 7400, 7406, 7408, 7415, 7423, 7426; 1 thin section.

\section{Synonymy.}

* 1850 Tetracoenia Dupiniana; Orbigny, (2), p. 121

1851 Tetracoenia Dupiniana; Orbigny, p. 166, Fig. 303

1987 Holocystis bucowinensis; Kuzmicheva, p. 222,

Pl. 1, Fig. 3

1988 Holocystis bukowinensis; Kuzmicheva \& Aliev,

p. 157 , Pl. 1, Fig. 4

v 2000 Nowakocoenia cieszynica; Kolodziej \& Gedl,

p. 187 , Figs. $3,8,9$

v 2006a Holocystis dupini; Löser, p. 291, Fig. 1d

v 2010 Holocystis dupini; Löser, p. 597, Fig. 3.10

\section{Dimensions.}

\begin{tabular}{cllcccc}
\hline $\mathbf{( 4 1 4 2 )}$ & \multicolumn{1}{c}{$\mathbf{n}$} & \multicolumn{1}{c}{$\boldsymbol{m i n}-\mathbf{m a x}$} & $\boldsymbol{\mu}$ & \multicolumn{1}{c}{$\mathbf{s}$} & $\mathbf{v}$ & $\boldsymbol{\mu} \pm \mathbf{s}$ \\
\hline $\mathrm{cl}$ & 20 & $1.45-1.86$ & 1.68 & 0.11 & 6.6 & $1.56-1.78$ \\
$\mathrm{ccd}$ & 20 & $2.18-3.53$ & 2.76 & 0.46 & 16.7 & $2.30-3.22$ \\
$\mathrm{~s}$ & $4+4+2.8$ & & & & & \\
\hline
\end{tabular}


Description. Plocoid colony. Calicular outline quadratic to circular. Septa compact. Septa and costae in cross section in the wall thick, thinner towards the centre. Symmetry of septa radial and regularly tetrameral. Cycles of septa regular. Septal cycles differ in length. Septa short. First septal cycle reaches $25 \%$ of the calicular diameter, further cycles are shorter. Septa free. Septal distal margin unknown, lateral face occasionally with medium size thorns. Pali or paliform lobes absent. Costae present, sub-confluent to non-confluent. Synapticulae absent. Columella absent. Endotheca consists of regular tabulae and occasional dissepiments. Wall present, compact, probably parathecal. Coenosteum medium broad (ca. $30 \%$ of the calicular diameter), consists of costae and tabulae. Budding extracalicinal.

Occurrence. (?) Early Hauterivian (Radiatus zone) of France (Yonne) Seignelay. Barremian of Azerbaijan (Kubatlinskij r.) Alikuliushagi; Turkmenistan (Krasnovodskaya obl.) Tuarkyr, Keldshe Mt. Early Barremian of Turkmenistan (Krasnovodskaya obl.) Small Balkans. Barremian (Moutoniceras - Giraudi zone) of France (Drôme) Serre de Bleyton. Late Barremian to Early Aptian (Lenticularis zone) of Mexico (Sonora) Municipio Ures, Cerro de Oro. Late Barremian to Early Aptian (Sarasini - Weissi zone) of Poland (Slaskie, Bielsko-
Biala) Bielsko-Biala, Rudzica. Early Aptian of France (Aube) Les Croûtes (?). Early Albian (Tardefurcata zone) of Spain (Cataluña, Tarragona) Com. Baix Penedés, Mun. Sant Martí Sarroca, Can Grau.

\section{DISCUSSION}

The corals come from six different layers within the section (Table 1), of which, only layer three to five have delivered a considerably high number of species. Layer three reaches, with 18 species, a normal number for a autochthon coral fauna. The various layers share many species; larger faunas show more correlation than smaller faunas (Fig. 7).

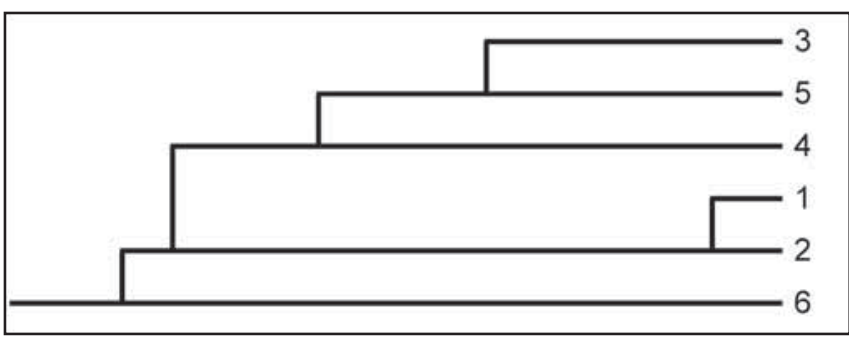

Figure 7. Correlation of the coral bearing layers based on the coral fauna. The Correlation Ratio coefficient was applied.

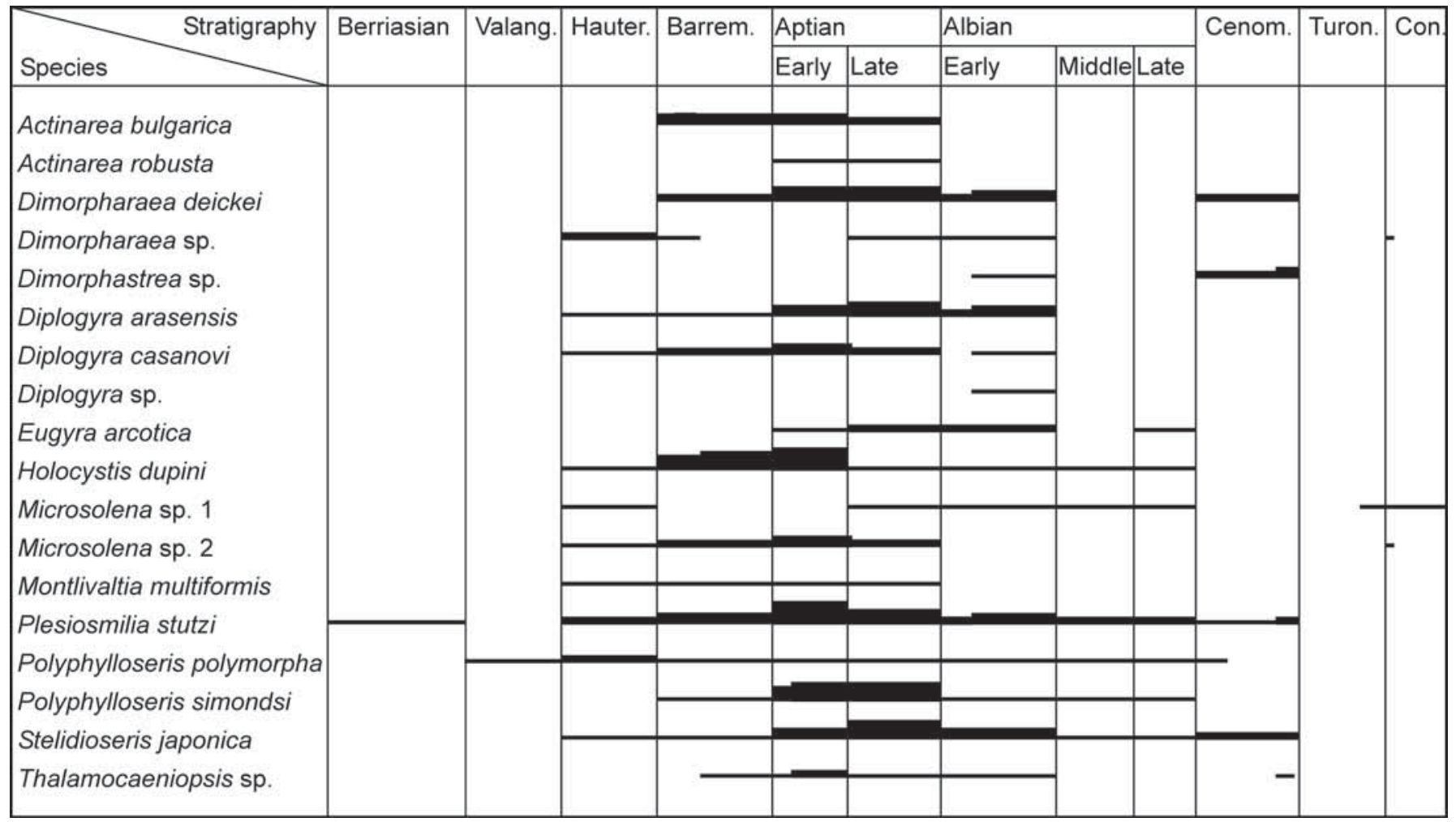

Figure 8. Stratigraphic distribution and commonness of species of the La Muela fauna. The thickness of the bars indicates the number of regions (not localities) in which the species concerned was found. The regions group localities together that have the same age and that are located in the same basin, in the same continental margin, or on the same intra oceanic platform. 
The fauna is represented by six suborders (out of eleven occurring in the Cretaceous; Löser, 2009). Corals of the suborder Microsolenina clearly dominate (42\%), followed by corals classified here in the Faviina suborder (32\%). The remaining suborders have low numbers of species. These distribution patterns are well comparable to faunas in a comparable facies (e.g., Löser, 2013a, fig. 13). All genera are common elements of Aptian faunas; the present fauna does not represent exotic, new or endemic elements. All genera present in the La Muela fauna have extremely long ranges; the shortest range from the Barremian to the Early Albian (Holocystis) or Hauterivian to the Albian (Diplogyra). The majority of the genera originates in the Jurassic and ranges into the early Late Cretaceous (see Löser, 2005 for details).

The coral association represents a typical combination of common species with ranges from the Hauterivian to the Early Albian. Only two species were found before the Hauterivian, but nine species show ranges beyond the Albian (Fig. 8). There are only three species that are found above the Cenomanian/Turonian boundary.

The palaebiogeographic relationships of the present fauna do not show much correlation, because of the low number of species. The fauna from the La Muela mountain ranges correlates mainly with faunas of a Late Barremian to Early Albian age, ranging palaeogeographically from the Western Hemisphere to the central Tethys (Fig. 9). Correlations with Hauterivian and Cenomanian faunas represent the long ranges that fossil coral species have.

\section{ACKNOWLEDGEMENTS}

We are grateful for the assistance of Cathleen Helbig (Munich, Germany) who did much preparatory work and Lucinda Puebla Clark (Hermosillo) who made most of the measurements of calicular dimensions. Financial support of a sabbatical UNAM/PASPA project for the first author is acknowledged. Grammatical correction was undertaken by Matthew Copley (Kingston, Surrey, UK). A review by Bernard Lathuilière (Nancy) helped to improve the article.

\section{REFERENCES}

Alloiteau, J. 1936. Sur la présence, dans le calcaire a Spatangues de la Haute-Marne, de Plesiosmilia villersensis Koby. Bulletin de la Société géologique de France, 5, 6, 507-510.

Alloiteau, J. 1946-47. Paléontologie. Anales de la Escuela de Peritos Agrícolas y de Especialidades Agropecuarias y de los Servicios Técnicos de Agricultura, 6, 187-243.

Alloiteau, J. 1952. Embranchement des coelentérés. In: Traité de Paléontologie (ed. Piveteau, J.). Masson, Paris, 1, 376-684.

Alloiteau, J. 1954. Sur cinq genres nouveaux de madréporaires post-paléozoïques. Bulletin de la Société géologique de France, 6, 3, 873-887.

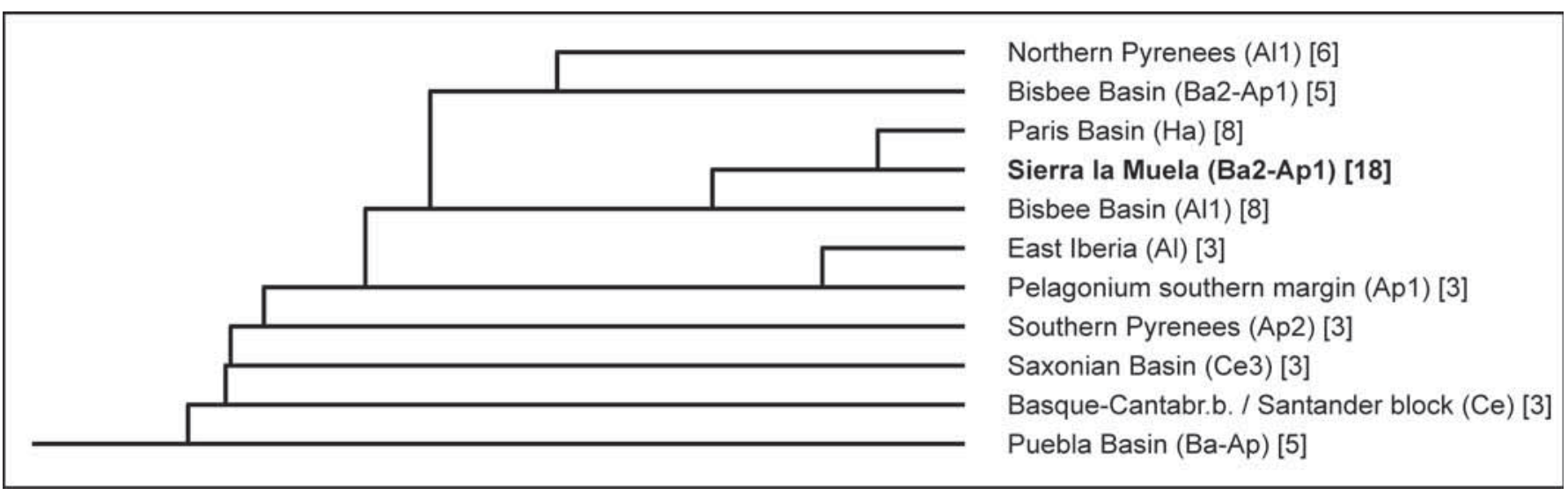

Figure 9. Correlation of provinces with joint species of the study area. Provinces with less than two joint species are suppressed. The Correlation Ratio coefficient was applied. For details of calculation see Löser \& Minor (2007). 
Angelis d'Ossat, G. de 1905. Coralli del Cretacico inferiore della Catalogna. Palaeontographia Italica, 9, 169-251.

Arnaud-Vanneau, A. \& Thieuloy, J.P. 1972. Orbitolinopsis buccifer n. sp. nouveau représentant du genre Orbitolinopsis Silvestri des calcaires urgoniens du Vercors (France). Revue de Micropaléontologie, 14, 4, 248-258.

Baron-Szabo, R.C. 1997. Die Korallenfazies der ostalpinen Kreide (Helvetikum, Allgäuer Schrattenkalk; Nördliche Kalkalpen, Brandenberger Gosau) Taxonomie, Palökologie. Zitteliana, 21, 3-97.

Baron-Szabo, R.C. \& González León, C.M. 1999. Lower Cretaceous corals and stratigraphy of the Bisbee Group (Cerro de Oro and Lampazos areas), Sonora, Mexico. Cretaceous Research, 20, 465-497.

Baron-Szabo, R.C. \& González León, C.M. 2003. Late Aptian-Early Albian corals from the Mural Limestone of the Bisbee Group (Tuape and Cerro de Oro areas), Sonora, Mexico. In: Bob F. Perkins Memorial Volume (ed. Scott, R.W.). Special Publications in Geology, 225, 187-225.

Baron-Szabo, R.C. \& Steuber, T. 1996. Korallen und Rudisten aus dem Apt im tertiären Flysch des Parnass-Gebirges bei Delphi-Arachowa. Berliner geowissenschaftliche Abhandlungen, (E) 18, 3-75.

Bataller, J. 1937. La fauna coral-lina del Cretàcic de Catalunya i regions limítrofes. Arxius de l'escola superior d'agricultura, (N.S.), 3, 1, 1-299.

Bataller, J. 1947. Sinopsis de las especies nuevas del Cretácico de España. Memorias de la Real Academia de Ciencias y Artes de Barcelona, 3, 28, 12, 279-392.

Bataller, J. 1949. Segundo suplemento a $<$ La fauna coral-lina del Cretàcic de Catalunya i regions limítrofes $>$. Anales de la Escuela de Peritos Agrícolas y superior de Agricultura y de los Servicios técnicos de Agricultura, 5, 3-58.

Bataller, J. 1959. Primer suplemento a la $<$ Sinopsis de las especies nuevas del Cretáceo de España $>$. Boletín del Instituto geológico y minero de España, 70, 1-77.

Blumenbach, J.F. 1805. Abbildungen naturhistorischer Gegenstände. Dieterich, Göttingen.

Bölsche, W. 1866. Die Korallen des norddeutschen Jura- und Kreide-Gebirges. Zeitschrift der Deutschen Geologischen Gesellschaft, 18, 439-486.

Bölsche, W. 1877. Über einige Korallen aus der westphälischen Kreide. Jahresbericht des naturwissenschaftlichen Vereins zu Osnabrück, 3, 68-71.

Bover Arnal, T., Löser, H., Moreno Bedmar, J.A., Salas, R. \& Strasser, A. 2012. Corals on the slope (Aptian, Maestrat Basin, Spain). Cretaceous Research, 37, 43-64.

Carreño, A.L., Perrilliat, M.C., Gonzáles-Arreola, C., Applegate, S.P., Carranza-Castañeda, O. \& Martinez Hernandez, E. 1989. Fosiles Tipo Mexicanos. Universidad Nacional Autónoma de México, Mexico City.

Dietrich, W.O. 1926. Steinkorallen des Malms und der Unterkreide im südlichen Deutsch-Ostafrika. Palaeontographica, (suppl.7), 1, 43-62.

Duncan, P.M. 1884. A revision of the families and genera of the sclerodermic Zoantharia Edwards et Haime, or Madreporaria (M. Rugosa excepted) (1-4). Journal of the Linnean Society of London, Zoology, 18, 104-105, 1-204.
Eguchi, M. 1936. Three new genera of corals from the Lower Cretaceous of Japan. Proceedings of the Imperial Academy of Japan, 12, 3, 70-72.

Eguchi, M. 1951. Mesozoic hexacorals from Japan. Science Reports of the Tohoku Imperial University, (2, Geology), 24, 1-96.

Felix, J. 1891. Versteinerungen aus der mexicanischen Jura und Kreideformation. Palaeontographica, 37, 140-194.

Felix, J. 1900. Über die Gruppe der Montlivaltiaceae. Sitzungsberichte der Naturforschenden Gesellschaft zu Leipzig, (February, 6 1900), 20-24.

Fourcade, E. 1970. Le Jurassique et le Crétacé aux confins des Chaînes Bètiques et Ibériques (Sud-Est de l'Espagne). $\mathrm{PhD}$ Thesis, Université de París (unpublished).

Fourcade, E., Jerez, L., Rodriguez-Estrella, T. \& Jaffrezo, M. 1972. El Jurásico terminal y el Cretácico inferior de la Sierra de La Muela (prov. de Murcia). Consideraciones sobre las biozonas con foraminíferos del AlbienseAptiense del SE de España. Revista Española de Micropaleontología, $\mathrm{N}^{\mathrm{o}}$ extraordinario. XXX aniversario E.N. Adaro, 215-248.

Fromentel, E. de 1857. Description des polypiers fossiles de l'étage Néocomien. Bulletin de la société des sciences historiques et naturelles de l'Yonne, 1-78.

Fromentel, E. de 1861. Introduction à l'étude des polypiers fossiles. Mémoires de la Société d'émulation du Doubs, $3,5,1.357$.

Götz, S., Löser, H. \& Schmid, D.U. 2005. Reef development on a deepening platform, two Early Cretaceous coralgal patch reefs (Catí, Llàcova Formation, eastern Spain) compared. Cretaceous Research, 26, 6, 864-881.

Jerez, L. 1973. Geología de la Zona Prebética en la transversal de Elche de la Sierra y sectores adyacentes (provincias de Albacete y Murcia). PhD Thesis, Universidad de Granada (unpublished).

Jerez, L. 1979a. Contribución a una nueva síntesis de las Cordilleras Béticas. Boletín Geológico y Minero, XC-VI, $1-53$.

Jerez, L. 1979b. Mapa Geológico Nacional de España E. 1/50.000 no 889 (24-35) Moratalla. Hoja y Memoria explicativa. I.G.M.E., Madrid.

Koby, F. 1896. Monographie des polypiers crétacés de la Suisse (1). Abhandlungen der Schweizerischen Paläontologischen Gesellschaft, 22, 1-28.

Koby, F. 1898. Monographie des polypiers crétacés de la Suisse (3). Abhandlungen der Schweizerischen Paläontologischen Gesellschaft, 24, 63-100.

Kolodziej, B. \& Gedl, E. 2000. Nowakocoenia cieszynica gen. et sp. nov. (Scleractinia) and its Barremian-Aptian age based on Dinocysts (Polish Outer Carpathians). Annales Societatis Geologorum Poloniae, 70, 181-192.

Kuzmicheva, E.I. 1987. [Corals from Lower Barremian organogenous buildups in the Malyy Balkhan and Tuarkyr.] In: Geologicheskoe stroenie Turkmenistana (ed. Amanniyazov, K.N.). Ylum, Aschabad, 217-262.

Kuzmicheva, E.I. \& Aliev, O.B. 1988. [Corals.] In: Cretaceous fauna of Azerbaijan (eds. Ali-Zade, A.A., Aliev, G.A. \& Aliev, M.M.). Elm, Baku, 153-184. 
Lamouroux, J.V.F. 1821. Exposition méthodique des genres de l'ordre des polypiers. Agasse, Paris.

Lonsdale, W. 1849. Notes on fossil Zoophytes found in the deposits described by Dr.Fitton in his Memoir entitled "A stratigraphical account of the section from Atherfield to Rocken End". Quarterly Journal of the Geological Society of London, 5, 55-103.

Löser, H. 1994. La faune corallienne du mont Kassenberg à Mülheim-sur-la-Ruhr (Bassin crétacé de Westphalie, Nord Ouest de l'Allemagne). Coral Research Bulletin, 3, 1-93.

Löser, H. 2004. PaleoTax - a database program for palaeontological data. Computer \& Geosciences, 30, 5, 513-521.

Löser, H. 2005. Stratigraphy of Cretaceous coral genera. Neues Jahrbuch für Geologie und Paläontologie, Abhandlungen, 238, 231-277.

Löser, H. 2006a. Taxonomy, stratigraphic distribution and palaeobiogeography of the Early Cretaceous coral genus Holocystis. Revista mexicana de ciencias geológicas, 23, 3, 288-301.

Löser, H. 2006b. Barremian corals from San Antonio Texcala, Puebla, Mexico - A review of the type material of Felix 1891. Boletín del Instituto Geológico de México, 114, $1-68$.

Löser, H. 2008. A new solitary coral genus of the suborder Heterocoeniina (Scleractinia) from the Aptian (Cretaceous) of Spain. Paläontologische Zeitschrift, 82, 3, 279-284.

Löser, H. 2009. Fossile Korallen aus Jura und Kreide. Aufbau, Klassifikation, Bestimmung und Fundmöglichkeiten. CPress Verlag, Dresden.

Löser, H. 2010. The Barremian coral fauna of the Serre de Bleyton mountain range (Drôme, France). Annalen des Naturhistorischen Museums in Wien, 112, 575-612.

Löser, H. 2012. Intraspecific variation in the genus Stelidioseris (family Actinastraeidae, suborder Archeocaeniina, order Scleractinia; Jurassic-Cretaceous). Geologica Belgica, $15,4,382-387$.

Löser, H. 2013a. An Early Albian shallow marine coral fauna from Southern France - insight into evolution and palaeobiogeography of Cretaceous corals. Palaeobiodiversity and Palaeoenvironments, 5, 1-43.

Löser, H. 2013b. Late Aptian (Cretaceous) corals from Central Greece. Neues Jahrbuch für Geologie und Paläontologie, Abhandlungen, 267, 1, 89-116.

Löser, H. \& Ferry, S. 2006. Coraux du Barrémien du Sud de la France (Ardèche et Drôme). Geobios, 39, 4, 469-489.

Löser, H. \& Minor, K. 2007. Palaeobiogeographic aspects of Late Barremian to Late Albian coral faunas from Northern Mexico (Sonora) and the southern USA (Arizona, Texas). Neues Jahrbuch für Geologie und Paläontologie, Abhandlungen, 245, 2, 193-218.

Löser, H. \& Raeder, M. 1995. Aptian/Albian coral assemblages of the Helicon Mountains (Boeotia, Greece), palaeontological, palaeoecological and palaeogeographical aspects. Coral Research Bulletin, 4, 37-63.

Löser, H. \& Saldaña-Villodre, J.C. 2008. Colonial corals from the Early Aptian siliciclastic Montlivaltia Marls of Jumilla (Murcia, Spain). Revista Española de Paleontología, 23, 1, 1-6.
Löser, H., Stemann, Th.A. \& Mitchell, S.F. 2009. Oldest Scleractinian fauna from Jamaica (Hauterivian, Benbow Inlier). Journal of Paleontology, 83, 3, 333-349.

Martín-Chivelet, J., Berasategui, X., Rosales, I., Vilas, L., Vera, J.A., Caus, E., Gráfe, K.U., Mas, R., Puig, C., Segura, M., Robles, S., Floquet, M., Quesada, S., Ruiz-Ortiz, P.A., Frenegal-Martínez, M.A., Salas, R., García, A., MartínAlgarra, A., Arias, C., Meléndez, M., Chacón, B., Molina, J.M., Sanz, J.L., Castro, J.M., García-Hernández, M., Carenas, B., García-Hidalgo, J., Gil, J. \& Ortega, F. 2002. Cretaceous. In: The Geology of Spain (eds. Gibbons, W. \& Moreno, T.). The Geological Society, London, 255-292.

Matthews, S.C. 1973. Notes on open nomenclature and on synonymy lists. Palaeontology, 16, 4, 713-719.

Milaschewitsch, C. 1876. Die Korallen der Nattheimer Schichten (2). Palaeontographica, 21, 62-123.

Moreno Bedmar, J.A., Ramírez, L., Company, M., Delanoy, G., Bover Arnal, T., Bulot, L., Latil, J.-L. \& Salas, R. 2009. Biostratigrafía de los amonites de Can Casanyes. Macizo de Garraf (Barcelona, España). Batalleria, 14, 91-98.

Morycowa, E. 1971. Hexacorallia et Octocorallia du Crétacé inférieur de Rarau (Carpathes orientales roumaines). Acta Palaeontologica Polonica, 16, 1/2, 1-149.

Morycowa, E. \& Marcopoulou-Diacantoni, A. 2002. Albian corals from the Subpelagonian zone of Central Greece (Agrostylia, Parnassos region). Annales Societatis Geologorum Poloniae, 72, 1-65.

Morycowa, E. \& Masse, J.P. 2009. Lower Cretaceous Microsolenina (Scleractinia) from Provence (Southern France). Annales Societatis Geologorum Poloniae, 79, 97-140.

Morycowa, E., Masse, J.P., Vilas, L. \& Arias, C. 2001. Montlivaltia multiformis Toula (Scleractinia) from the Aptian of the Prebetic domain (SE Spain). Revista Española de Paleontología, 16, 1, 131-144.

Morycowa, E. \& Roniewicz, E. 1995. Microstructural disparity between Recent fungiine and Mesozoic microsolenine scleractinians. Acta Palaeontologica Polonica, 40, 4, 361-385.

Orbigny, A. de 1849. Note sur les polypiers fossiles. Masson, Paris.

Orbigny, A. de 1850. Prodrôme de Paléontologie stratigraphique universelle des animaux mollusques et rayonnés (1-2). Masson, Paris.

Orbigny, A. de 1851. Cours élémentaire de Paléontologie (3:) Polypiers ou Zoophytes. Masson, Paris.

Reig Oriol, J. 1994. El género Eugyra en el Cretáceo del nordeste español. Batalleria, 4, 31-36.

Reyeros Navarro, M.M. 1963. Corales del Cretacico inferior de San Juan Raya, Estado de Puebla. Paleontologia mexicana, 17, 1-21.

Rodriguez-Estrella, T. 1979. Geología e hidrogeología del sector de Alcaraz-Liétor-Yeste (prov. de Albacete). Síntesis geológica de la Zona Prebética. Tesis doctoral. Memorias Instituto Geológico y Minero de España, 97.

Roniewicz, E. 1966. Les madréporaires du Jurassique supérieur de la bordure des monts de Sainte-croix, Pologne. Acta Palaeontologica Polonica, 11, 2, 157-264. 
Ruiz-Ortiz, P.A., Arias, C., Castro, J.M., De Gea, G. A., Nieto, L.M. \& Vilas, L. 2010. Early Cretaceous shallowwater carbonate platform development in the Southern Iberian Continental Paleomargin: Considerations about the westernmost Prebetic. 18th International Sedimentological Congress. Mendoza, Argentina, Abstracts, p. 771.

Schöllhorn, E. 1998. Geologie und Paläontologie des Oberapt im Becken von Organyà (Nordspanien). Coral Research Bulletin, 6, 1-139.

Sikharulidze, G.Ya. 1985. [Hexacorals from the Urgonian facies of the Dzirul Massif and its northern frame.]. Trudy Akademija Nauk Gruzinskoj SSR, Geologiceskij Institut, 59, 1-110.

Silvestri, A. 1931. Foraminiferi del Cretaceo della Somalia. Palaeontographia Italica, 32, 143-204.

Stoliczka, F. 1873. The corals or Anthozoa from the Cretaceous rocks of South India. Memoirs of the Geological Survey of India, Palaeontologia Indica, 4, 8, 4/5, 130-202.

Tomás, S., Löser, H. \& Salas Roig, R. 2008. Low-light and nutrient-rich coral assemblages in an Upper Aptian carbonate platform of the southern Maestrat Basin (Iberian Chain, eastern Spain). Cretaceous Research, 29, 509-534.

Tomes, R.F. 1893. Observations on the affinities of the genus Astrocoenia. Quarterly Journal of the Geological Society of London, 49, 569-573.

Toula, F. 1877. Geologische Untersuchungen im westlichen Theile des Balkans und der angrenzenden Gebiete (4:) Ein geologisches Profil von Osmanich am Arcer, über den Sveti-Nicola-Balkan, nach Ak-Palanka an der Nisava. Sitzungsberichte der Mathematisch-Naturwissenschaftliche Classe der Kaiserlichen Akademie der Wissenschaften, 1, 75, 465-549.

Toula, F. 1889. Geologische Untersuchungen im centralen Balkan. Denkschriften der Kaiserlichen Akademie der Wissenschaften, Mathematisch-Physikalische Klasse, 55, 1-108.

Turnšek, D. \& Buser, S. 1974. Spodnjekredne korale, hidrozoji in hetetide $\mathrm{z}$ Banjske Planote in Trnovskega Gozda. Razprave Slovenska akademija znanosti in umetnosti, 4, 17, 2, 81-124.

Turnšek, D. \& Mihajlovic, M. 1981. Lower Cretaceous Cnidarians from eastern Serbia. Razprave Slovenska akademija znanosti in umetnosti, 4, 23, 1, 1-54.

Vaughan, T.W. 1905. A critical review of the literature of the simple genera of Fungida, with a tentative classification. Proceedings of the United States National Museum, 28, 1401, 371-424.
Vaughan, T.W. \& Wells, J.W. 1943. Revision of the suborders, families and genera of Scleractinia. Special Papers. Geological Society of America, 44, 1-363.

Vera, J.A. (Coord.), Arias, C., García-Hernández, M., LópezGarrido, A.C., Martín-Algarra, A., Martín-Chivelet, J., Molina, J.M., Rivas, P., Ruiz-Ortiz, P.A., Sanz de Galdeano, C. \& Vilas, L. 2004. Las Zonas externas Béticas y el Paleomargen Sudibérico. In: Geología de España (ed. Vera, J.A.). SGE-IGME, Madrid, 354-361.

Verrill, A.E. 1868-70. Notes on the Radiata in the museum of Yale College, with descriptions of new genera and species. No. 6. Review of the corals and polyps of the West Coast of America. Transactions of the Connecticut Academy of Arts and Sciences, 1, 2, 377-567.

Vilas, L., Arias, C., Castro, J.M., de Gea, G.A. \& RuizOrtiz, P.A. 2009. The Aptian tectosedimentary episode in the Easthern Prebetic (SE Spain). 8th International Symposium on the Cretaceous System, Abstract volume, p. $120-121$.

Vilas, L., Dabrio, C.J., Peláez, J.R. \& García Hernández, M. 2001. Dominios sedimentarios generados durante el período extensional Cretácico inferior entre Cazorla y Hellín (Béticas externas). Su implicación en la estructura actual. Revista de la Sociedad Geologica de España, 14 (1-2), 113-122.

Vilas, L., Martín-Chivelet, J. \& Arias, C. 2003. Integration of subsidence and sequence stratigraphic analyses in the Cretaceous carbonate platforms of the Prebetic (Jumilla-Yecla Region), Spain. Palaeogeography, Palaeoclimatology, Palaeoecology, 200, 107-129.

Vilas, L., Martín-Chivelet, J., Arias, C., Giménez, R., RuizOrtiz, P.A. Castro, J.M. Masse, J.P. \& Estévez, A. 1998. Cretaceous carbonate platforms of the Spanish Levante. Sedimentary evolution and sequence stratigraphy. 15th IAS International Sedimentological Congress, Field trip Guide Book, p. 293-315.

Vilas, L., Masse, J.P., \& Arias, C. 1993. Aptian mixed terrigenous and carbonate platforms from Iberic and Prebetic regions (Spain). In: Cretaceous Carbonate Platforms. American Association Petroleum Geology, Tulsa, 243-253.

Wells, J.W. 1932. Corals of the Trinity Group of the Commanchean of central Texas. Journal of Paleontology, 6, 3, 225-256.

Wells, J.W. 1956. Scleractinia. In: Treatise on Invertebrate Paleontology (ed. Moore, R.C.). University Press of Kansas, Lawrence, Kan., F328-F444. 
